\title{
Adaptive Control of Double-Gimbal Control-Moment Gyro with Unbalanced Rotor
}

\author{
Jasim Ahmed* \\ Robert Bosch Corporation, Palo Alto, California 94303 \\ and \\ Dennis S. Bernstein ${ }^{\dagger}$ \\ University of Michigan, Ann Arbor, Michigan 48109-2140
}

\begin{abstract}
A double-gimbalcontrol-moment gyro (CMG) is modeled using Lagrange's equations, and an adaptive feedback control law is developed to follow a commanded CMG motion. The control law does not require knowledge of the mass properties of the CMG. A Lyapunov argument is used to prove that command following is achieved globally with asymptotic convergence. Numerical simulationsare performed to illustrate the command following algorithm. A CMG testbed was designed and constructed to implement and demonstrate the adaptive algorithm. This testbed is described, and experimental results are given.
\end{abstract}

\section{Introduction}

A CTUATORS for spacecraft fall into two distinct categories, namely, inertial and noninertial. Inertial actuators provide torques to a spacecraft by reacting against inertial space and, thus, change the angular momentum of the spacecraft. Thrusters, which require fuel, are the principal type of inertial actuators. Magnetic actuators, which react to the Earth's magnetic field and which do not require fuel, also serve as inertial actuators.

Noninertial actuators include reaction wheels, momentum wheels, and single-gimbal and double-gimbal control moment gyros (CMGs). Whereas these actuators require electric power, they do not require fuel, and they do not change the total angular momentum of the spacecraft. ${ }^{1}$ Among noninertial actuators, the dual-axis CMG is the most sophisticated because it has the ability to apply control torques around arbitrary axes with the exception of singular orientations corresponding to gimbal lock. In applications, multiple CMGs are used for reliability, to avoid gimbal lock, and to avoid large angle motion.

Because a CMG is a multibody system, its dynamics are complex, especially if large angle motion is allowed. Theoretical analysis of CMG dynamics is given in Ref. 2, whereas engineering aspects of CMG controlare considered in Refs. 3-5. In the present paper we are concerned with the problem of wheel imbalance in CMGs. At high rotational speeds $(5,000-10,000 \mathrm{rpm}$ is typical for CMGs), small mass imbalances in the CMG wheel or due to asymmetric lubricant buildup can produce significant disturbance forces at the frequency of the wheel angular velocity. ${ }^{6}$ Mass imbalance effects also arise in momentum wheels where active isolation stages are used for disturbance suppression? These studies suggest that noninertial actuators are often the source of the largest component of disturbance forces on spacecraft.

In the present paper we model a double-gimbal CMG with unknown mass imbalance, mounted on a support structure fixed to the Earth. Using Lagrange's equations (see Ref. 8), we account for large angle motion and the presence of imbalance, which to the best of our knowledge has not been done. A double-gimbal CMG testbed was designed and constructed to permit various control experiments to

Received 26 June 2000; revision received 10 April 2001; accepted for publication 13 April 2001. Copyright (C) 2001 by the American Institute of Aeronautics and Astronautics, Inc. All rights reserved. Copies of this paper may be made for personal or internal use, on condition that the copier pay the $\$ 10.00$ per-copy fee to the Copyright Clearance Center, Inc., 222 Rosewood Drive, Danvers, MA 01923; include the code 0731-5090/02 \$10.00 in correspondence with the CCC.

*Systems Engineer, Research and Technology Center, 4009 Miranda Avenue; jasim.ahmed@rtc.bosch.com.

${ }^{\dagger}$ Professor, Department of Aerospace Engineering; dsbaero@engin. umich.edu. be performed. The control objective is to develop a control law that counteracts the effects of mass imbalance without knowledge of the mass distribution of the CMG while allowing the CMG to follow a commanded motion that includes unbounded rotational motion of the CMG gimbals and wheel.

Adaptive control laws for mechanical systems with linear dependence on parameters that are able to follow a commanded motion have been developed..$^{-11}$ However, in Ref. 9 and 10, the class of commanded motions do not include those that permit unbounded rotational motion of the mechanical systems because the rotational motion of the system is described in terms of angles and their derivatives. In Ref. 11, differential geometric techniques are used to guarantee convergence to a set consisting of four states, one of which is the desired state, but requires knowledge of bounds of the unknown parameters.

In this paper, the rotational motion of the CMG is described by using trigonometric functions of the half-angles of the gimbals and wheel, which transform unbounded CMG rotational motion to motion on a compact set. This formulation permits the development of a control algorithm, which is presented in Sec. IV, that allows unbounded rotational motion of the CMG gimbals and wheel. A proof of the control law is based on a variant of standard Lyapunov arguments found in Ref. 12 to account for the motion on closed sets. The control law is a direct adaptive control law ${ }^{13-16}$ and has the form of a dynamic compensator whose order depends on the number of uncertain parameters and whose states provide estimates of the wheel and gimbal moments of inertia and centers of mass. Our controller can be viewed as an extension of the control law derived in Ref. 17 for spacecraft tracking with unknown mass distribution.

The contents of the paper are as follows. In Sec. II, we describe the equations that govern the CMG motion; in Sec. III, we define the command following problem; and in Sec. IV, we formulate a control algorithm that permits the CMG to track a desired trajectory. In Sec. V, we illustrate the adaptive control law using a numerical example, in Sec. VI, we describe the experimental setup, and in Sec. VII, we present experimental results.

\section{Equations of Motion}

In this section, we model the CMG as a system of interconnected rigid bodies and apply Lagrange's equations for a system of rigid bodies to obtain the equations that govern the CMG's motion.

For a system of rigid bodies, Lagrange's equations are given by

$$
\frac{\mathrm{d}}{\mathrm{d} t}\left(\frac{\partial L}{\partial \dot{q}_{i}}\right)-\frac{\partial L}{\partial q_{i}}=Q_{i}^{\prime}, \quad i=1, \ldots, n
$$

where $n$ is the number of degrees of freedom, $q_{1}, \ldots, q_{n} \in \Re$ are the independent generalized coordinates, $\dot{q}_{1}, \ldots, \dot{q}_{n} \in \Re$ are the derivatives of $q_{1}, \ldots, q_{n}$, and $L=L(q, \dot{q}) \in \Re$ is the Lagrangian of the 


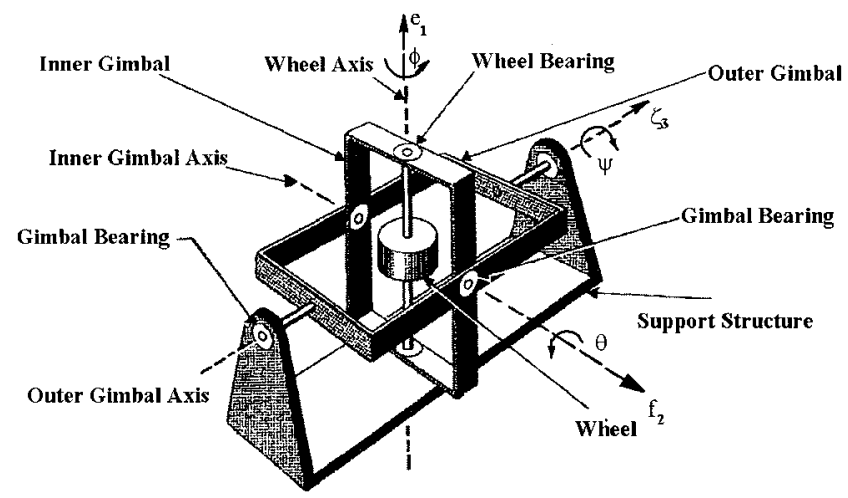

Fig. 1 CMG nomenclature.

system, where $q=\left[q_{1} \cdots q_{n}\right]^{T} \in \Re^{n}$ and $\dot{q}=\left[\dot{q}_{1} \cdots \dot{q}_{n}\right]^{T} \in \Re^{n}$, and $L$ is given by

$$
L=T-\mathcal{V}
$$

where $T=T(q, \dot{q}) \in \Re$ is the kinetic energy of the system, $\mathcal{V}=$ $\mathcal{V}(q) \in \mathfrak{R}$ is the potential energy of the system, $Q_{1}^{\prime}, \ldots, Q_{n}^{\prime} \in \mathfrak{R}$ are the generalized forces not derivable from a potential function and are given by

$$
Q_{i}^{\prime}=\sum_{j=1}^{m} F_{j} \cdot \frac{\partial \rho_{j}}{\partial q_{i}}, \quad i=1, \ldots, n
$$

where, for $j=1, \ldots, m, F_{j}$ is a force not derivable from a potential function and acting at position $\rho_{j}=\rho_{j}(q)$ and $m$ is a positive integer representing the total number of forces not derivable from a potential. If there are no forces not derivable from a potential function, then $Q_{1}^{\prime}=\cdots=Q_{n}^{\prime}=0$.

The kinetic energy of a single rigid body $r$ is

$$
T=\frac{1}{2} \omega_{r} \cdot I_{x}^{r} \cdot \omega_{r}+\frac{1}{2} m_{r} v_{x} \cdot v_{x}+m_{r} \omega_{r} \cdot \rho_{x y} \times v_{x}
$$

where $\frac{1}{2} \omega_{r} \cdot I_{x}^{r} \cdot \omega_{r}$ represents the rotational kinetic energy and $\frac{1}{2} m_{r} v_{x} \cdot v_{x}+m_{r} \omega_{r} \cdot \rho_{x y} \times v_{x}$ represents the translational kinetic energy, where $m_{r}>0$ is the mass of $r, x$ is a point on $r, y$ is the center of mass of $r, \rho_{x y}=\rho_{x y}(q)$ is the vector from $x$ to $y, v_{x}=v_{x}(q, \dot{q})$ is the velocity of $x, \omega_{r}=\omega_{r}(q, \dot{q})$ is the angular velocity of $r$, and $I_{x}^{r}=I_{x}^{r}(q)$ is the positive definite inertia tensor of $r$ about $x$. The potential energy of $r$ in the presence of a uniform gravitationalfield is

$$
\mathcal{V}=-m_{r} g \cdot \rho_{y}
$$

where $\rho_{y}=\rho_{y}(q)$ denotes the position of $y$ and $g$ is the gravity vector.

The CMG shown in Fig. 1 consists of a rectangular outer gimbal, which rotates through an angle $\psi$ about an axis $\zeta_{3}$, an inner gimbal, which rotates within the outer gimbal through an angle $\theta$ about an the outer gimbal, and a wheel motor, which is attached to the inner gimbal.

We model the outer gimbal, the inner gimbal, and the wheel as rigid bodies and assume that the support structure on which the CMG is mounted is inertially fixed. We employ the following body-fixed frames to determine the kinetic energy of the CMG. Let $\left(\zeta_{1}, \zeta_{2}, \zeta_{3}\right)$ be a frame fixed to the outer gimbal, where $\zeta_{3}$ is the outer gimbal axis and $\zeta_{2}$ is the inner gimbal axis. Let $\left(f_{1}, f_{2}, f_{3}\right)$ be a frame fixed to the inner gimbal, where $f_{1}$ is the wheel axis and is obtained by rotating $\left(\zeta_{1}, \zeta_{2}, \zeta_{3}\right)$ through an angle $\theta$ about $\zeta_{2}$ so that

$$
\left[\begin{array}{l}
f_{1} \\
f_{2} \\
f_{3}
\end{array}\right]=\left[\begin{array}{ccc}
\cos \theta & 0 & -\sin \theta \\
0 & 1 & 0 \\
\sin \theta & 0 & \cos \theta
\end{array}\right]\left[\begin{array}{l}
\zeta_{1} \\
\zeta_{2} \\
\zeta_{3}
\end{array}\right]
$$

Finally, let $\left(e_{1}, e_{2}, e_{3}\right)$ be a frame fixed to the wheel obtained by rotating $\left(f_{1}, f_{2}, f_{3}\right)$ through an angle $\phi$ about $f_{1}$ so that

$$
\left[\begin{array}{l}
e_{1} \\
e_{2} \\
e_{3}
\end{array}\right]=\left[\begin{array}{ccc}
1 & 0 & 0 \\
0 & \cos \phi & \sin \phi \\
0 & -\sin \phi & \cos \phi
\end{array}\right]\left[\begin{array}{l}
f_{1} \\
f_{2} \\
f_{3}
\end{array}\right]
$$

Now, the kinetic energy of the CMG is the sum of the kinetic energy of the outer gimbal, the inner gimbal, and the wheel. When Eq. (4) is applied to the outer gimbal, inner gimbal, and wheel, the kinetic energy of the CMG is given by

$$
T=\frac{1}{2} \dot{q}^{T} M(q) \dot{q}
$$

where $q=\left[\begin{array}{lll}\phi & \theta & \psi\end{array}\right]^{T} \in \mathfrak{R}^{3}, \dot{q}=\left[\begin{array}{lll}\dot{\phi} & \dot{\theta} & \dot{\psi}\end{array}\right]^{T} \in \mathfrak{R}^{3}$, and $M: \mathfrak{R}^{3} \rightarrow$ $\Re^{3 \times 3}$ is defined by

$$
\begin{aligned}
& M(q)=G_{w r}^{T}(q) I_{a}^{w} G_{w r}(q)+G_{w t}^{T}(q) G_{w t}(q)+G_{i r}^{T}(q) I_{b}^{i} G_{i r}(q) \\
& +G_{i t}^{T}(q) G_{i t}(q)+G_{o}^{T}(q) I_{c}^{o} G_{o}(q)
\end{aligned}
$$

where $a, b$, and $c$ are arbitrary points on the axis of rotation of the wheel, inner gimbal, and outer gimbal, respectively; $I_{c}^{o}$ is the inertia matrix of the outer gimbal about $c$ expressed in frame $\left(\zeta_{1}, \zeta_{2}, \zeta_{3}\right) ; I_{b}^{i}$ is the inertia matrix of the inner gimbal about $b$ expressed in frame $\left(f_{1}, f_{2}, f_{3}\right)$; and $I_{a}^{w}$ is the inertia matrix of the wheel about the point a expressed in frame $\left(e_{1}, e_{2}, e_{3}\right)$, where $G_{o}: \mathfrak{R}^{3} \rightarrow \mathfrak{R}^{3 \times 3}, G_{i r}: \mathfrak{R}^{3} \rightarrow \mathfrak{R}^{3 \times 3}$, $G_{i t}: \mathfrak{R}^{3} \rightarrow \mathfrak{R}^{3 \times 3}, G_{w r}: \mathfrak{R}^{3} \rightarrow \mathfrak{R}^{3 \times 3}$, and $G_{w t}: \mathfrak{R}^{3} \rightarrow \mathfrak{R}^{3 \times 3}$ are defined by

$$
\begin{gathered}
G_{o}(q) \triangleq\left[\begin{array}{lll}
0 & 0 & 0 \\
0 & 0 & 0 \\
0 & 0 & 1
\end{array}\right], \quad G_{i r}(q) \triangleq\left[\begin{array}{ccc}
0 & 0 & -\sin \theta \\
0 & 1 & 0 \\
0 & 0 & \cos \theta
\end{array}\right] \\
G_{w r}(q) \triangleq\left[\begin{array}{ccc}
1 & 0 & -\sin \theta \\
0 & \cos \phi & \cos \theta \sin \phi \\
0 & -\sin \phi & \cos \theta \cos \phi
\end{array}\right]
\end{gathered}
$$

where

$$
\begin{gathered}
G_{i t}(q) \triangleq\left[\begin{array}{ccc}
0 & 0 & 0 \\
0 & 0 & m_{i}\left(f_{2} \times \rho_{b u}\right) \cdot\left(\zeta_{3} \times \rho_{c b}\right) \\
0 & m_{i}\left(f_{2} \times \rho_{b u}\right) \cdot\left(\zeta_{3} \times \rho_{c b}\right) & m_{i}\left[\left(\zeta_{3} \times \rho_{c b}\right) \cdot\left(\zeta_{3} \times \rho_{c b}\right)+2\left(\zeta_{3} \times \rho_{b u}\right) \cdot\left(\zeta_{3} \times \rho_{c b}\right)\right]
\end{array}\right] \\
G_{w t}(q) \triangleq\left[\begin{array}{ccc}
0 & m_{w}\left(e_{1} \times \rho_{a t}\right) \cdot\left(f_{2} \times \rho_{b a}\right) & m_{w}\left(e_{1} \times \rho_{a t}\right) \cdot\left(\zeta_{3} \times \rho_{c a}\right) \\
m_{w}\left(e_{1} \times \rho_{a t}\right) \cdot\left(f_{2} \times \rho_{b a}\right) & Z_{1} & Z_{2} \\
m_{w}\left(e_{1} \times \rho_{a t}\right) \cdot\left(\zeta_{3} \times \rho_{c a}\right) & Z_{2} & Z_{3}
\end{array}\right]
\end{gathered}
$$

axis $f_{2}$ perpendicular to the outer gimbal axis, and a wheel fixed to the inner gimbal, which spins through an angle $\phi$ about an axis $e_{1}$ perpendicular to the inner gimbal axis. We assume that the $\mathrm{CMG}$ is constructed so that $\zeta_{3}$ is perpendicular to $e_{1}$ initially. The CMG has three actuators, specifically, an outer gimbal motor, which is fixed to the support structure, an inner gimbal motor, which is fixed to

$$
\begin{aligned}
Z_{1}= & m_{w}\left[\left(f_{2} \times \rho_{b a}\right) \cdot\left(f_{2} \times \rho_{b a}\right)+2\left(f_{2} \times \rho_{a t}\right) \times\left(f_{2} \times \rho_{b a}\right)\right] \\
Z_{2}= & m_{w}\left[\left(\zeta_{3} \times \rho_{c a}\right) \cdot\left(f_{2} \times \rho_{b a}\right)+\left(\zeta_{3} \times \rho_{a t}\right) \cdot\left(f_{2} \times \rho_{b a}\right)\right. \\
& \left.+\left(f_{2} \times \rho_{a t}\right) \cdot\left(\zeta_{3} \times \rho_{c a}\right)\right] \\
Z_{3}= & m_{w}\left[\left(\zeta_{3} \times \rho_{c a}\right) \cdot\left(\zeta_{3} \times \rho_{c a}\right)+2\left(\zeta_{3} \times \rho_{a t}\right) \cdot\left(\zeta_{3} \times \rho_{c a}\right)\right]
\end{aligned}
$$


where $t$ and $u$ are the centers of mass of the wheel and inner gimbal, respectively. The potential energy of the CMG is the sum of the potentialenergy of the outer gimbal, the inner gimbal, and the wheel. We assume that the gravitational field is uniform and when Eq. (5) is applied, the potential energy of the CMG is given by

$$
\mathcal{V}(q) \triangleq-g \cdot\left(m_{w} \rho_{t}+m_{i} \rho_{u}+m_{o} \rho_{v}\right)
$$

where $v$ is the center of mass of the outer gimbal. The generalized forces not derivable from a potential, obtained by applying Eq. (3), are

$$
\begin{gathered}
Q_{\phi}^{\prime}=\tau_{w}+f_{w}+s_{w} \\
Q_{\theta}^{\prime}=\tau_{i}+f_{i}+s_{i} \\
Q_{\psi}^{\prime}=\tau_{o}+f_{o}+s_{o}
\end{gathered}
$$

where $\tau_{w}, \tau_{i}$, and $\tau_{o}$ are the torques applied by the wheel, the inner gimbal, and the outer gimbal motor, respectively; $f_{w}, f_{i}$, and $f_{o}$ are the torques due to friction; and $s_{w}, s_{i}$, and $s_{o}$ are the torques due to stiffness acting on the wheel, the inner gimbal, and the outer gimbal, respectively.For the CMG described in Sec. VI, the stiffness torques model the effect of the cables on the CMG.

Applying Eq. (1), we obtain

$$
M(q) \ddot{q}+[C(q, \dot{q})-F(q, \dot{q})] \dot{q}+G(q)-S(q)=u
$$

where $C: \mathfrak{R}^{3} \times \mathfrak{R}^{3} \rightarrow \mathfrak{R}^{3 \times 3}$ is defined by

$$
C(q, \dot{q}) \triangleq \frac{1}{2}\left[\dot{M}(q, \dot{q})+B^{T}(\dot{q}, q)-B(\dot{q}, q)\right]
$$

$\dot{M}: \mathfrak{R}^{3} \times \mathfrak{R}^{3} \rightarrow \mathfrak{R}^{3 \times 3}$ is defined by

$$
\dot{M}(q, \dot{q}) \triangleq \frac{\partial M^{T}}{\partial q}\left(\dot{q} \otimes I_{3}\right)
$$

$I_{n}$ is the $n$ by $n$ identity matrix, $\otimes$ is the Kronecker product, $\left(\partial M^{T} / \partial q\right): \mathfrak{R}^{3} \times \mathfrak{R}^{3} \rightarrow \mathfrak{R}^{3 \times 9}$ is defined by

$$
\frac{\partial M^{T}}{\partial q} \triangleq\left[\frac{\partial M}{\partial \phi} \frac{\partial M}{\partial \theta} \frac{\partial M}{\partial \psi}\right]
$$

$B: \mathfrak{R}^{3} \times \mathfrak{R}^{3} \rightarrow \mathfrak{R}^{3 \times 3}$ is defined by

$$
B(q, \dot{q}) \triangleq\left(I_{3} \otimes \dot{q}^{T}\right) \frac{\partial M}{\partial q}
$$

$G: \mathfrak{R}^{3} \rightarrow \mathfrak{R}^{3}$ is defined by states and express the command following problem in terms of these error coordinates.

Consider the transformation $h: \mathfrak{R}^{3} \rightarrow \mathfrak{R}^{6}$ given by

$$
h(p) \triangleq\left[\begin{array}{c}
\sin p_{1} / 2 \\
\sin p_{2} / 2 \\
\sin p_{3} / 2 \\
\cos p_{1} / 2 \\
\cos p_{2} / 2 \\
\cos p_{3} / 2
\end{array}\right]
$$

where $p=\left[\begin{array}{lll}p_{1} & p_{2} & p_{3}\end{array}\right]^{T}$. We observe from Eq. (25) that

$$
h\left(\Re^{3}\right)=U
$$

where $U$ is the compact set given by

$$
U \triangleq\left\{\left(w_{1}, w_{2}, w_{3}, w_{4}, w_{5}, w_{6}\right) \in \mathfrak{R}^{6}: w_{i}^{2}+w_{i+3}^{2}=1, i=1,2,3\right\}
$$

Let

$$
z_{d}=h\left(q_{d}\right)
$$

where $q_{d}=\left[\begin{array}{lll}\phi_{d} & \theta_{d} & \psi_{d}\end{array}\right]^{T}:[0, \infty) \rightarrow \mathfrak{R}^{3}$ is the commanded motion. Using Eqs. (26) and (28), we observe that $z_{d}$ is bounded for every $q_{d}$, including those $q_{d}$ that are unbounded. Thus, unbounded rotational commanded motion of the CMG is transformed to motion on the compact set $U$.

Next, we show that Eq. (18) can be rewritten in terms of $z$, where

$$
z=h(q)
$$

The dependence of $M$ on $q$ is only in the form of trigonometric functions of $\phi, \theta$, and $\psi$. Because $\sin \phi=2 \sin \phi / 2 \cos \phi / 2$ and $\cos \phi=\sin ^{2} \phi / 2-\cos ^{2} \phi / 2$, with similar expressions for $\theta$ and $\psi$, it follows that $M(q)$ can be rewritten in terms of $z$ to obtain the function $\hat{M}(z)$. Similarly, because the dependence of $C$ and $G$ on $q$ is only in the form of the trigonometric functions, we can express $C(q, \dot{q})$ and $G(q)$ in terms of $z$ and $\dot{q}$ to obtain the functions $\hat{C}(z, \dot{q})$ and $\hat{G}(z)$. Assuming the arguments of $F$ and $S$ depend only on trigonometric functions, we rewrite $F$ and $S$ to obtain $\hat{F}(z, \dot{q})$ and $\hat{S}(z)$.

Rewriting Eq. (18) in terms of $z$ and $\dot{q}$, we obtain

$$
\hat{M}(z) \ddot{q}+[\hat{C}(z, \dot{q})-\hat{F}(z, \dot{q})] \dot{q}+\hat{G}(z)-\hat{S}(z)=u
$$

$$
G(q) \triangleq\left[\begin{array}{c}
-m_{w} g \cdot\left(e_{1} \times \rho_{a t}\right) \\
-g \cdot\left[m_{w} \zeta_{3} \times\left(\rho_{c b}+\rho_{b a}+\rho_{a t}\right)+m_{i} \zeta_{3} \times\left(\rho_{c b}+\rho_{b u}\right)+m_{o} \zeta_{3} \times \rho_{c v}\right]
\end{array}\right]
$$

and $u \triangleq\left[\begin{array}{lll}\tau_{w} & \tau_{i} & \tau_{o}\end{array}\right]^{T}$. In addition, we assume the friction and stiffness torques are of the form

$$
\left[\begin{array}{c}
f_{w} \\
f_{i} \\
f_{o}
\end{array}\right]=F(q, \dot{q}) \dot{q}, \quad\left[\begin{array}{c}
s_{w} \\
s_{i} \\
s_{o}
\end{array}\right]=S(q)
$$

where $F: \mathfrak{R}^{3} \times \mathfrak{R}^{3} \rightarrow \mathfrak{R}^{3 \times 3}$ and $S: \mathfrak{R}^{3} \rightarrow \mathfrak{R}^{3}$.

\section{Error Equations Command Following Problem}

In this section, we employ a suitable change of coordinates so that unbounded commanded rotational motion of the CMG is transformed to motion on a compact set. We then define suitable error

$$
\dot{z}=\left[\begin{array}{cc}
0 & O(\dot{q}) \\
-O(\dot{q}) & 0
\end{array}\right] z
$$

where $O: \Re^{3} \rightarrow \Re^{3 \times 3}$ is defined by

$$
O(p)=\frac{1}{2}\left[\begin{array}{ccc}
p_{1} & 0 & 0 \\
0 & p_{2} & 0 \\
0 & 0 & p_{3}
\end{array}\right]
$$

where $p=\left[\begin{array}{lll}p_{1} & p_{2} & p_{3}\end{array}\right]^{T}$.

Next, the error state $E_{z}$ is defined by

$$
E_{z} \triangleq\left[\begin{array}{c}
E \\
\hat{E}
\end{array}\right] \triangleq H\left(z_{d}\right) z
$$


where $E \in \mathfrak{R}^{3}, \hat{E} \in \mathfrak{R}^{3}$, and $H: \mathfrak{R}^{6} \rightarrow \mathfrak{R}^{6 \times 6}$ is given by

$$
H(w) \triangleq\left[\begin{array}{cccccc}
w_{4} & 0 & 0 & -w_{1} & 0 & 0 \\
0 & w_{5} & 0 & 0 & -w_{2} & 0 \\
0 & 0 & w_{6} & 0 & 0 & -w_{3} \\
w_{1} & 0 & 0 & w_{4} & 0 & 0 \\
0 & w_{2} & 0 & 0 & w_{5} & 0 \\
0 & 0 & w_{3} & 0 & 0 & w_{6}
\end{array}\right]
$$

where $w=\left[\begin{array}{llllll}w_{1} & w_{2} & w_{3} & w_{4} & w_{5} & w_{6}\end{array}\right]^{T}$. Using Eq. (34), we observe that that $H(w) H^{T}(w)=H^{T}(w) H(w)=I_{6}$ for all $w \in U$, where $U$ is given by Eq. (27). Hence, Eq. (33) implies

$$
z=H^{T}\left(z_{d}\right) E_{z}
$$

$$
E=\left[\begin{array}{c}
\sin \left(\phi / 2-\phi_{d} / 2\right) \\
\sin \left(\theta / 2-\theta_{d} / 2\right) \\
\sin \left(\psi / 2-\psi_{d} / 2\right)
\end{array}\right], \quad \hat{E}=\left[\begin{array}{c}
\cos \left(\phi / 2-\phi_{d} / 2\right) \\
\cos \left(\theta / 2-\theta_{d} / 2\right) \\
\cos \left(\psi / 2-\psi_{d} / 2\right)
\end{array}\right]
$$

Note that $E=0$ if and only if $\phi-\phi_{d}=0 \bmod 2 \pi, \theta-\theta_{d}=0 \bmod$ $2 \pi$, and $\phi-\phi_{d}=0 \bmod 2 \pi$. Furthermore, define the error state

$$
e_{\dot{q}} \triangleq \dot{q}-\dot{q}_{d}
$$

For the command following problem, assume $q_{d}:[0, \infty) \rightarrow \mathfrak{R}^{3}$ is $C^{2}$. Find a dynamic feedback control law of the form

$$
\begin{aligned}
\dot{\hat{\alpha}} & =f\left(z_{d}, \dot{q}_{d}, \ddot{q}_{d}, \hat{\alpha}, z, \dot{q}\right) \\
u & =g\left(z_{d}, \dot{q}_{d}, \ddot{q}_{d}, \hat{\alpha}, z, \dot{q}\right)
\end{aligned}
$$

for Eqs. (30) and (31), where $\hat{\alpha}(t) \in \mathfrak{R}^{v}, t \in[0, \infty)$, such that $E \rightarrow 0$ and $e_{\dot{q}} \rightarrow 0$ as $t \rightarrow \infty$ for all initial conditions $z(0) \in U, \dot{q}(0) \in \Re^{3}$, and $\hat{\alpha}(0) \in \mathfrak{R}^{\nu}$.

Command following problem as stated requires $E \rightarrow 0$ and $e_{\dot{q}} \rightarrow 0$, which using Eqs. (36) and (37) implies that the CMG follow a commanded motion, and permits all sufficiently smooth $q_{d}$, including those that are unbounded. Note that the control algorithm as stated in Eqs. (38) and (39) does not have to be independent of the mass distribution of the CMG. However, in Sec. IV we shall develop a control algorithm that requires no knowledge of the mass or inertia properties of the CMG.

Next, we recast Eqs. (30) and (31) in terms of the error states $E_{z}$ and $e_{\dot{q}}$ and restate the command following problem in terms of $E_{z}$ and $e_{\dot{q}}$. To do this, define $\tilde{M}:[0, \infty) \times \mathfrak{R}^{6} \rightarrow \mathfrak{R}^{3 \times 3}$ by

$$
\tilde{M}\left(t, E_{z}\right) \triangleq \hat{M}\left\{H^{T}\left[z_{d}(t)\right] E_{z}\right\}
$$

$\tilde{C}:[0, \infty) \times \mathfrak{R}^{6} \times \mathfrak{R}^{3} \rightarrow \mathfrak{R}^{3 \times 3}$ by

$$
\tilde{C}\left(t, E_{z}, e_{\dot{q}}\right) \triangleq \hat{C}\left\{H^{T}\left[z_{d}(t)\right] E_{z}, e_{\dot{q}}+\dot{q}_{d}(t)\right\}
$$

and $\tilde{G}:[0, \infty) \times \mathfrak{R}^{6} \rightarrow \mathfrak{R}^{3}$ by

$$
G\left(t, E_{z}\right) \triangleq \hat{G}\left\{H^{T}\left[z_{d}(t)\right] E_{z}\right\}
$$

When Eqs. (35) and (37) are used, Eqs. (30) and (31) become

$$
\begin{aligned}
& \tilde{M}\left(t, E_{z}\right)\left(\dot{e}_{\dot{q}}+\ddot{q}_{d}\right)=\left[-\tilde{C}\left(t, E_{z}, e_{\dot{q}}\right)+\tilde{F}\left(t, E_{z}, e_{\dot{q}}\right)\right]\left(e_{\dot{q}}+\dot{q}_{d}\right) \\
& \quad+\tilde{S}\left(t, E_{z}\right)-\tilde{G}\left(t, E_{z}\right)+u \\
& \dot{E}_{z}=\left[\begin{array}{cc}
0 & O\left(e_{\dot{q}}\right) \\
-O\left(e_{\dot{q}}\right) & 0
\end{array}\right] E_{z}
\end{aligned}
$$

Then, Eqs. (33) and (34) imply that

$$
E_{i}^{2}+\hat{E}_{i}^{2}=z_{i}^{2}+z_{i+3}^{2}, \quad i=1,2,3
$$

It follows from Eq. (45) that the command following problem is solved if and only if $E \rightarrow 0$ and $e_{\dot{q}} \rightarrow 0$ in Eqs. (43) and (44) for all initial conditions $E_{z} \in U$ and $e_{\dot{q}}(0) \in \Re^{3}$.

We assume that measurements of $q$ and $\dot{q}$ are available. It can be seen from Eqs. (25), (34), and (37) that the quantities $z, z_{d}, E, \hat{E}$, and $e_{\dot{q}}$ can be calculated. In Sec. IV, the control law Eqs. (38) and (39) is expressed in terms of $\hat{\alpha}, E_{z}, e_{\dot{q}}, z_{d}, \dot{q}_{d}$, and $\ddot{q}_{d}$.

\section{Adaptive Control Law}

In this section, we present a feedback control law that asymptotically follows a commanded trajectory. The control law does not require knowledge of the mass distribution of the CMG.

Using Eqs. (9), (12), (13), (34), and (40), we observethat $\tilde{M}\left(t, E_{z}\right)$ dependson the inertia, mass, and center of mass location parameters, namely, $I_{a 11}^{w}, I_{a 22}^{w}, I_{a 33}^{w}, I_{a 23}^{w}, I_{a 13}^{w}, I_{a 12}^{w}, I_{b 11}^{i}, I_{b 22}^{i}, I_{b 33}^{i}, I_{b 23}^{i}, I_{b 13}^{i}, I_{b 12}^{i}$, $I_{c 33}^{o}, m_{w}, m_{i}, m_{o}, \rho_{a t 1}, \rho_{a t 2}, \rho_{a t 3}, \rho_{b u 1}, \rho_{b u 2}, \rho_{b u 3}, \rho_{b a 1}, \rho_{b a 2}, \rho_{b a 3}$, $\rho_{c b 1}, \rho_{c b 2}$, and $\rho_{c b 3}$, where $\rho_{a t}$ is expressed in $\left(e_{1}, e_{2}, e_{3}\right), \rho_{b u}$ and $\rho_{b a}$ are expressed in $\left(f_{1}, f_{2}, f_{3}\right)$, and $\rho_{c b}$ is expressed in $\left(\zeta_{1}, \zeta_{2}, \zeta_{3}\right)$, and where $x_{i}$ is the $i$ th component of $x \in \Re^{n}$ and $A_{i j}$ is the $(i, j)$ entry of $A \in \Re^{m \times n}$. Next, note from Eqs. (9), (12), (13), (34), and (40) that $\tilde{M}\left(t, E_{z}\right)$ depends linearly on $\alpha_{m}$, where $\alpha_{m}$ consists of inertia, mass and center of mass location parameters, and products of these. In practice, some of these parameters may be known. In this case, we assume that $\alpha_{m}$ consists only of uncertain parameters and products of parameters, at least one of which is uncertain. It can be shown that the dimension of $\alpha_{m}$ is between 0 (no uncertain parameters) and 50 (all uncertain parameters).

Similarly, using Eqs. (23), (34), and (42), we observe that $\tilde{G}\left(t, E_{z}\right)$ depends on the gravitational parameters, namely, $g_{1}, g_{2}, g_{3}, m_{w}$, $m_{i}, m_{o}, \rho_{a t 1}, \rho_{a t 2}, \rho_{a t 3}, \rho_{b u 1}, \rho_{b u 2}, \rho_{b u 3}, \rho_{b a 1}, \rho_{b a 2}, \rho_{b a 3}, \rho_{c b 1}, \rho_{c b 2}$, $\rho_{c b 3}, \rho_{c v 1}$, and $\rho_{c v 2}$ where $g$ is expressed in an arbitrary inertially fixed frame and $\rho_{c v}$ is expressed in $\left(\zeta_{1}, \zeta_{2}, \zeta_{3}\right)$. Next, note from Eqs. (23), (34), and (42) that $\tilde{G}\left(t, E_{z}\right)$ depends linearly on $\alpha_{g}$, where $\alpha_{g}$ consists of the center of gravity location parameters and products of these. It can be shown that the dimension of $\alpha_{g}$ is between 0 (no uncertain parameters) and 15 (all uncertain parameters). For the friction and stiffness torques, we assume that there exist parameters $\alpha_{f}$ and $\alpha_{s}$, so that $\tilde{F}\left(t, E_{z}, e_{\dot{q}}\right)$ and $\tilde{S}\left(t, E_{z}\right)$ depend linearly on $\alpha_{f}$ and $\alpha_{s}$.

The number of uncertain parameters $v$ depends on assumptions made about the CMG configuration, as well as on $\tilde{F}$ and $\tilde{S}$. In the specialcase in which there are no friction and stiffness torques, there exists a common point that lies on the axis of rotation of all of these motors, that is, $a=b=c$ so that $G_{w t}=G_{i t}=0$, and $g_{2}=g_{3}=0$, where $g$ is expressed in an inertially fixed frame $\left(\epsilon_{1}, \epsilon_{2}, \epsilon_{3}\right)$ such that, at $t=0,\left(\epsilon_{1}, \epsilon_{2}, \epsilon_{3}\right)$ coincides with $\left(\zeta_{1}, \zeta_{2}, \zeta_{3}\right)$; then it can be seen that $v=21$. This is the case considered in Sec. V.

The following lemmas will be needed.

Lemma 1: $\hat{M}(z)$ and $\tilde{M}\left(t, E_{z}\right)$ are positive definite for all $z \in \Re^{6}$, $t \in[0, \infty)$, and $E_{z} \in \Re^{6}$.

Proof: Recall that $\hat{M}(z)$ is formed by replacing the trigonometric functions of the angles $\psi, \theta$, and $\phi$ by $z$ using Eq. (25). When $\hat{G}_{o}(z), \hat{G}_{i r}(z), \hat{G}_{i t}(z), \hat{G}_{w r}(z)$, and $\hat{G}_{w t}(z)$ are defined in a similar manner, it follows from Eqs. (9) and (29) that

$$
\begin{aligned}
\hat{M}(z) & =\hat{G}_{o}^{T}(z) I_{c}^{o} \hat{G}_{o}(z)+\hat{G}_{i r}^{T}(z) I_{b}^{i} \hat{G}_{i r}(z)+\hat{G}_{i t}^{T}(z) \hat{G}_{i t}(z) \\
& +\hat{G}_{w r}^{T}(z) I_{a}^{w} \hat{G}_{w r}(z)+\hat{G}_{w t}^{T}(z) \hat{G}_{w t}(z)
\end{aligned}
$$

From Eq. (46), it follows that $\hat{M}(z)$ is the sum of positive semidefinite terms and is, thus, positive semidefinite for all $z \in \Re^{6}$. Let $z \in \Re^{6}$ and let $p \in \mathfrak{R}^{3}$ satisfy $p^{T} \hat{M}(z) p=0$. Thus, it follows that

$$
\hat{G}_{o}(z) p=\hat{G}_{i r}(z) p=\hat{G}_{w r}(z) p=0
$$

When Eq. (10) is used, $\hat{G}_{o}(z) p=0$ implies that $p_{3}=0$. Similarly, $p_{3}=0$ and $\hat{G}_{i r}(z) p=0$ imply $p_{2}=0$. Finally, when Eq. (11) is used with $p_{2}=p_{3}=0$ and $\hat{G}_{w r}(z) p=0$, it follows that $p_{1}=0$; hence, $p=0$, which implies that $\hat{M}(z)$ is positive definite for all $z \in \mathfrak{R}^{6}$. Finally it follows from Eq. (40) that $\tilde{M}\left(t, E_{z}\right)$ is positive definite for all $t \in[0, \infty)$ and $E_{z} \in \Re^{6}$. 
Lemma 2: There exist $\mu_{1}>0$ and $\mu_{2}>0$ such that

$$
\begin{gathered}
\mu_{1} I_{3} \leq \hat{M}(z) \leq \mu_{2} I_{3}, \quad z \in U \\
\mu_{1} I_{3} \leq \tilde{M}\left(t, E_{z}\right) \leq \mu_{2} I_{3}, \quad t \in[0, \infty), \quad E_{z} \in U
\end{gathered}
$$

where $U$ is given by Eq. (27).

Proof: Because $U$ is a compact subset of $\Re^{6}, \hat{M}$ is a continuous function, and $\hat{M}(z)$ is positive definite for all $z \in \mathfrak{R}^{6}$, it follows that there exist positive $\mu_{1}$ and $\mu_{2}$ satisfying Eq. (48). Equation (49) is immediate.

Finally, we isolate the parameters that characterize the inertia, mass, center of mass locations, center of gravity locations, and the friction and stiffness torques by defining $Y:[0, \infty) \times \mathfrak{R}^{6} \times \mathfrak{R}^{3} \times$ $\mathfrak{R}^{3} \times \mathfrak{R}^{3} \times \mathfrak{R}^{3} \rightarrow \mathfrak{R}^{3 \times v}$ by

$$
\begin{aligned}
& Y\left(t, E_{z}, e_{\dot{q}}, \kappa, \tilde{\kappa}, \hat{\kappa}\right) \alpha \triangleq-\tilde{M}\left(t, E_{z}\right) \kappa-\tilde{C}\left(t, E_{z}, e_{\dot{q}}\right) \tilde{\kappa} \\
& +\tilde{F}\left(t, E_{z}, e_{\dot{q}}\right) \hat{\kappa}-\tilde{G}\left(t, E_{z}\right)+\tilde{S}\left(t, E_{z}\right)
\end{aligned}
$$

where $\kappa \in \mathfrak{R}^{3}, \tilde{\kappa} \in \mathfrak{R}^{3}, \hat{\kappa} \in \mathfrak{R}^{3}$, and $\alpha \in \mathfrak{R}^{v}$ is the vector of parameters.

Next, we present a control law that solves the command following problem with a proof based on Lyapunov theory. Note from the definition of the command following problem as stated in Sec. III that we are only interested in initial conditions that belong to the closed set $\mathfrak{R}^{3} \times U \times \mathfrak{R}^{\nu}$. The standard Lyapunov theorem as found in Ref. 18 is only valid for open sets, and so we use a variant of the standard Lyapunov argument found in Ref. 12.

Theorem: Assume that $\dot{q}_{d}$ and $\ddot{q}_{d}$ are bounded for all $t \in[0, \infty)$. Let $\Lambda:[0, \infty) \rightarrow \mathfrak{R}^{3 \times 3}$ be continuous, $K:[0, \infty) \rightarrow \mathfrak{R}^{3 \times 3}$ be continuous, $\Lambda_{1} \in \mathfrak{R}^{3 \times 3}, \Lambda_{2} \in \mathfrak{R}^{3 \times 3}, K_{1} \in \mathfrak{R}^{3 \times 3}$, and $K_{2} \in \mathfrak{R}^{3 \times 3}$ be such that $\Lambda_{1}, \Lambda_{2}$, and $\Lambda(t)$ are diagonal for all $t \in[0, \infty)$,

$$
\begin{array}{ll}
0<\Lambda_{1}<\Lambda(t)<\Lambda_{2}, & t \in[0, \infty) \\
0<K_{1}<K(t)<K_{2}, & t \in[0, \infty)
\end{array}
$$

Let $P \in \mathfrak{R}^{3 \times 3}$ be diagonal and positive definite, and let $Q \in \mathfrak{R}^{\nu \times v}$ be positive definite. Then the control law

$$
\begin{aligned}
\dot{\hat{\alpha}}= & Q^{-1} Y^{T}\left[t, E_{z}, e_{\dot{q}}, \ddot{q}_{d}-\Lambda O\left(e_{\dot{q}}\right) \hat{E},-\Lambda E\right. \\
& \left.+\dot{q}_{d}, e_{\dot{q}}+\dot{q}_{d}\right]\left(e_{\dot{q}}+\Lambda E\right) \\
u= & -Y\left[t, E_{z}, e_{\dot{q}}, \ddot{q}_{d}-\Lambda O\left(e_{\dot{q}}\right) \hat{E},-\Lambda E+\dot{q}_{d}, e_{\dot{q}}+\dot{q}_{d}\right] \hat{\alpha} \\
& -P E-K\left(e_{\dot{q}}+\Lambda E\right)
\end{aligned}
$$

solves the command following problem. Furthermore, $\hat{\alpha}$ is bounded for all $t \geq 0$, and $\dot{\hat{\alpha}} \rightarrow 0$ as $t \rightarrow \infty$.

Proof: Define $\sigma, \hat{e}, e_{z}$, and $\beta$ by

$$
\begin{gathered}
\sigma \triangleq e_{\dot{q}}+\Lambda E \\
\hat{e} \triangleq \hat{E}-\eta_{1} \\
e_{z} \triangleq\left[\begin{array}{ll}
E^{T} & \hat{e}^{T}
\end{array}\right]^{T} \\
\beta \triangleq \alpha-\hat{\alpha}
\end{gathered}
$$

where $\eta_{1}=\left[\begin{array}{lll}1 & 1 & 1\end{array}\right]^{T}$. For conciseness, we write

$$
\begin{aligned}
Y= & Y\left[t, e_{z}+\eta_{2}, \sigma-\Lambda E, \ddot{q}_{d}-\Lambda O(\sigma-\Lambda E)\left(\hat{e}+\eta_{1}\right),\right. \\
& \left.-\Lambda E+\dot{q}_{d}, \sigma-\Lambda E+\dot{q}_{d}\right]
\end{aligned}
$$

where $\eta_{2}=\left[\begin{array}{llllll}0 & 0 & 0 & 1 & 1 & 1\end{array}\right]^{T}$. Using Eqs. (43), (44), and (53-59), we obtain

$$
\begin{gathered}
\tilde{M}\left(t, e_{z}+\eta_{2}\right) \dot{\sigma}=Y \beta-\tilde{C}\left(t, e_{z}+\eta_{2}, \Delta E\right) \sigma-P E-K \sigma \\
\dot{e}_{z}=\left[\begin{array}{cc}
0 & O(\sigma-\Lambda E) \\
-O(\sigma-\Lambda E) & 0
\end{array}\right]\left(e_{z}+\eta_{2}\right) \\
\dot{\beta}=-Q^{-1} Y^{T} \sigma
\end{gathered}
$$

Let $\chi=\left[\sigma^{T} e_{z}^{T} \beta^{T}\right]^{T}$. Then the origin $\chi=0$ is an equilibrium solution of the system (60-62).

Next, we show that $\sigma \rightarrow 0$ and $E \rightarrow 0$ as $t \rightarrow \infty$ for initial conditions $\chi(0) \in D$, where $D \triangleq \Re^{3} \times \hat{U} \times \Re^{v}$, where $\hat{U} \triangleq$ $\left\{w \in \mathfrak{R}^{6}: w+\eta_{2} \in U\right\}$. To do this we use Theorem 3.2 of Ref. 12, which entails constructing a Lyapunov function, showing that $D$ is an invariant set and all solutions are bounded.

Consider the candidate Lyapunov function $V:[0, \infty) \times \mathfrak{R}^{3} \times$ $\Re^{6} \times \Re^{v} \rightarrow \Re$ defined by

$$
V(t, \chi)=\frac{1}{2}\left[\sigma^{T} \tilde{M}\left(t, e_{z}+\eta_{2}\right) \sigma+\beta^{T} Q \beta\right]+E^{T} P E+\hat{e}^{T} P \hat{e}
$$

The candidate Lyapunov function is the sum of a pseudokinetic energy term $\frac{1}{2} \sigma^{T} M\left(t, e_{z}+\eta_{2}\right) \sigma$, a pseudopotential energy term $E^{T} P E+\hat{e}^{T} P \hat{e}$, and $\frac{1}{2} \beta^{T} Q \beta$, a positive definite function in the parameter error. The total time derivative of $V$ along the trajectories of the system is given by

$$
\dot{V}(t, \chi)=-\sigma^{T} K(t) \sigma-E^{T} P \Lambda(t) E \leq-\sigma^{T} K_{1} \sigma-E^{T} P \Lambda_{1} E
$$

Because $D$ is closed, $V$ is positive semidefinite on $[0, \infty) \times D$, and $\dot{V}$ satisfies Eq. (64), it follows that $V$ is a valid Lyapunov function on $D$.

Next we show that $D$ is an invariant set and that all solutions are bounded. Let $\chi(t)$ be a solution of Eqs. (60-62) defined on an interval $I$, such that $\chi(0) \in D$. Now $\chi(t)$ remains in $D$ because $e_{z, i}^{2}(t)+\left[e_{z, i+3}(t)+1\right]^{2}=1, t \in I$. When Eq. (49) and $E_{z}=e_{z}+\eta_{2}$ are used, it follows that

$$
\mu_{1} I_{3} \leq \tilde{M}\left(t, e_{z}+\eta_{2}\right) \leq \mu_{2} I_{3}, \quad t \in[0, \infty), \quad e_{z} \in \hat{U}
$$

Now, it follows from Eq. (65) that

$$
W_{1}(\chi) \leq V(t, \chi) \leq W_{2}(\chi), \quad t \in[0, \infty), \quad \chi \in D
$$

where $W_{1}: \Re^{3} \times \Re^{6} \times \Re^{v} \rightarrow \Re$ and $W_{2}: \Re^{3} \times \Re^{6} \times \Re^{v} \rightarrow \Re$ are the radially unbounded positive definite functions

$$
\begin{aligned}
& W_{1}(\chi)=\frac{1}{2}\left[\mu_{1} \sigma^{T} \sigma+\beta^{T} Q \beta\right]+E^{T} P E+\hat{e}^{T} P \hat{e} \\
& W_{2}(\chi)=\frac{1}{2}\left[\mu_{2} \sigma^{T} \sigma+\beta^{T} Q \beta\right]+E^{T} P E+\hat{e}^{T} P \hat{e}
\end{aligned}
$$

When the sets $A_{i, \delta}=\left\{\chi \in D: W_{i}(\chi) \leq \delta\right\}$ are defined, where $i=1,2, \delta>0$, and $\Omega_{t, \delta}=\{\chi \in D: V(t, \chi) \leq \delta\}$, where $t \geq 0$ and $\delta>0$, it follows that

$$
A_{2, \delta} \subset \Omega_{t, \delta} \subset A_{1, \delta}, \quad t \geq 0, \quad \delta>0
$$

Because $W_{1}$ and $W_{2}$ are radially unbounded, it follows that the sets $A_{1, \delta}$ and $A_{2, \delta}$ are bounded for all $\delta>0$, and, furthermore, there exists $\hat{\delta}>0$ large enough such that $\chi(0) \in A_{2, \hat{\delta}}$. Now from Eq. (64) it follows that $V[t, \chi(t)]$ is not increasing and with the use of Eq. (69) that the solution $\chi(t)$ remains in the compact set $A_{1, \hat{\delta}}$. It now follows from Theorem 2.4 of Ref. 18 that $\chi(t)$ exists for all $t \geq 0$.

Next note that the total time derivative of $W_{3}: \mathfrak{R}^{3} \times \Re^{6} \times \Re^{v} \rightarrow \mathfrak{R}$ defined by

$$
W_{3}(\chi)=-\sigma^{T} K_{1} \sigma-E^{T} P \Lambda_{1} E
$$

along the trajectories of the system is given by

$$
\begin{aligned}
& \dot{W}_{3}(t, \chi)=-2 \sigma^{T} K_{1}\left[\tilde{M}\left(t, e_{z}+\eta_{2}\right)\right]^{-1}[Y \beta-E-K \sigma \\
& \left.\quad-\tilde{C}\left(t, e_{z}+\eta_{2}, \sigma-\Lambda E\right)\right]-2 E^{T} \Lambda_{1} F(\sigma-\Lambda E)\left(e_{z}+\eta_{2}\right)
\end{aligned}
$$

Because $\tilde{M}^{-1}, \tilde{C}, Y, F, \chi, \dot{q}_{d}$, and $\ddot{q}_{d}$ are continuous functions, $\chi(t)$ is bounded, and by assumption $\Lambda, K, \dot{q}_{d}$, and $\ddot{q}_{d}$ are bounded, it follows that $W_{3}[t, \chi(t)]$ is bounded.Using Theorem 3.2 of Ref. 12, we conclude that $\sigma \rightarrow 0$ and $E \rightarrow 0$. Furthermore, because $\dot{q}_{d}$ and $\ddot{q}_{d}$ are bounded and $\sigma \rightarrow 0$ and $E \rightarrow 0$, it follows from Eq. (62) that $\dot{\beta} \rightarrow 0$ and, thus, $\dot{\hat{\alpha}} \rightarrow 0$.

Because $\sigma \rightarrow 0, E \rightarrow 0$, and $\Lambda$ is bounded, it follows from Eq. (55) that $e_{\dot{q}} \rightarrow 0$. Hence, we conclude that Eqs. (53) and (54) solve the command following problem. 


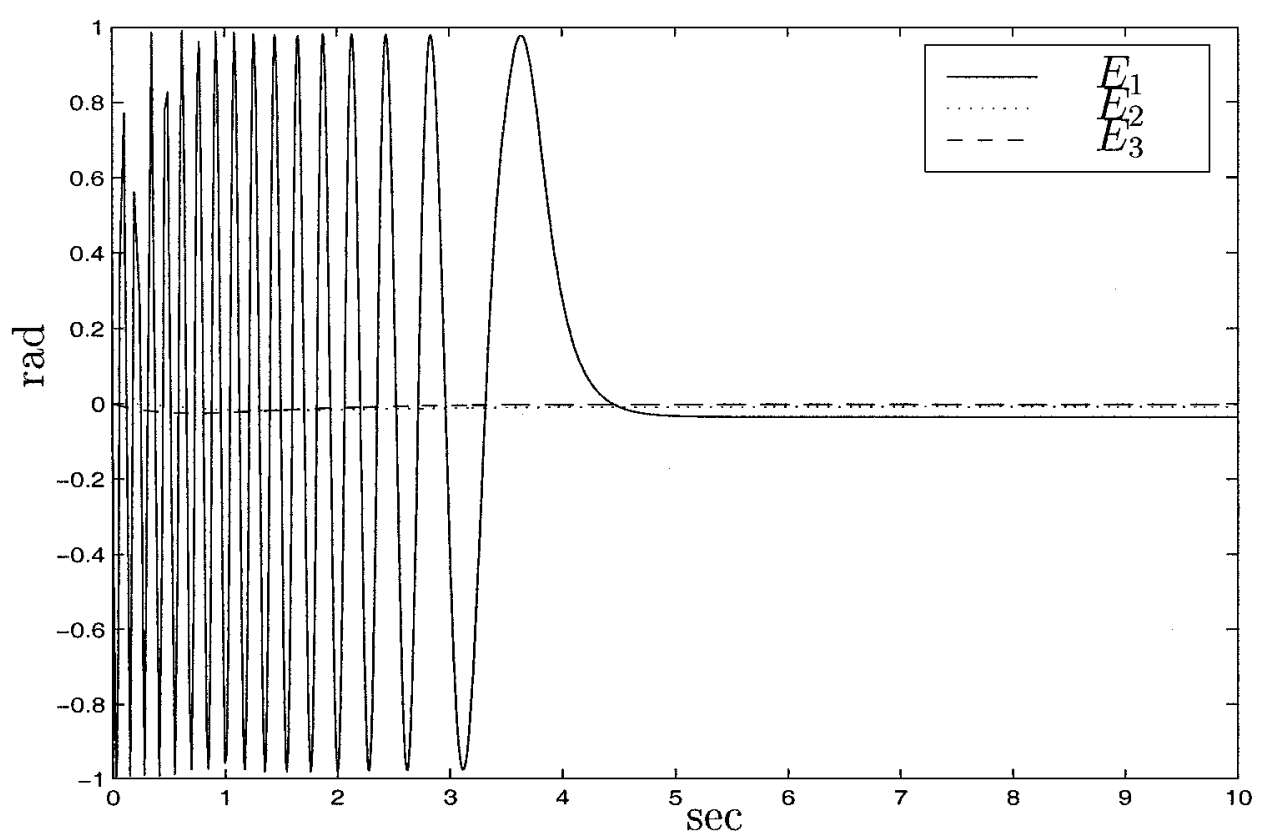

Fig. 2 Error states $E_{1}, E_{2}$, and $E_{3}$.

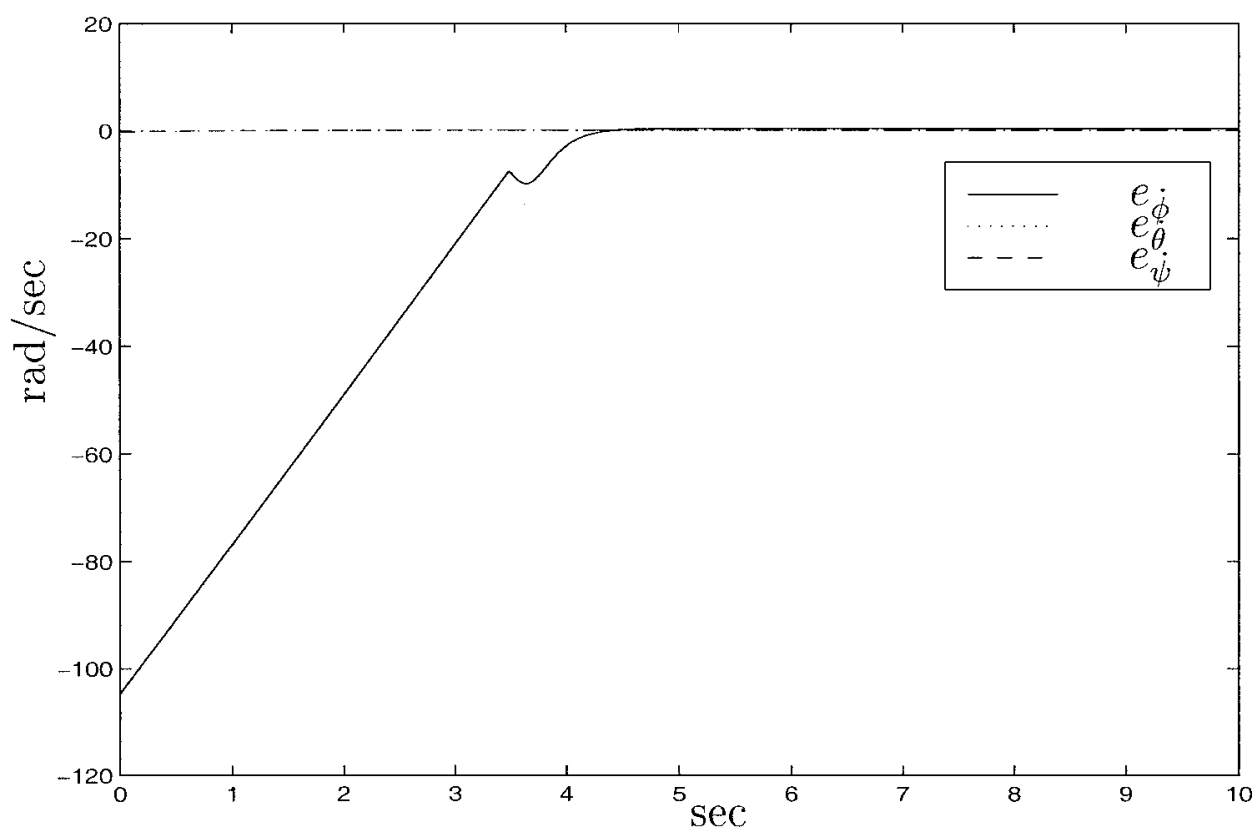

Fig. 3 Error states $e_{\dot{q}}=\left[\begin{array}{lll}e_{\phi} & e_{\dot{\theta}} & e_{\dot{\psi}}\end{array}\right]^{T}$.

Using Eqs. (50), (53), and (54), we observe that the control algorithm does not require any knowledge of the mass distribution of the CMG and only requires knowledge of the CMG states $z, \dot{q}, z_{d}, \dot{q}_{d}$, and $\ddot{q}_{d}$. Furthermore, we observe that the right-hand side of Eq. (53) is independent of $\hat{\alpha}$ and that the right-hand side of Eq. (54) is dependent only on the CMG states and $\hat{\alpha}$. Hence, the control law (53) and (54) is a proportiona-integral compensator.

The parameter $Q$ represents the gain of the adaptation law, and $\Lambda, K$, and $P$ represent the gains of the proportional-integral controller. In Sec. VII, we describe how we chose these gains for our experimental setup. The state $\hat{\alpha}$ represents adjustable parameters, whereas Eq. (53) represents the mechanism for adjusting these parameters. Although the time derivative of the adaptive parameter $\hat{\alpha}$ converges to zero as $t \rightarrow \infty, \hat{\alpha}$ does not necessarily converge. See Ref. 17 for additional details concerning the use of $\hat{\alpha}$ for parameter identification.

\section{Numerical Example}

In this section we illustrate command following for the desired trajectory:

$$
\begin{gathered}
\phi_{d}(t)=2000 \pi / 60 t \mathrm{rad} \\
\theta_{d}(t)=(30 \pi / 180) \sin (15 \pi / 180 t) \mathrm{rad} \\
\psi_{d}(t)=(40 \pi / 180) \sin (10 \pi / 180 t) \mathrm{rad}
\end{gathered}
$$

This command representsa CMG motion in which the wheel spins at a constant rate of $1000 \mathrm{rpm}$, the inner gimbal oscillates sinusoidally with an amplitude of $30 \mathrm{deg}$ and frequency of $15 \mathrm{deg} / \mathrm{s}$, and the outer gimbal oscillates sinusoidally with an amplitude of $40 \mathrm{deg}$ and a frequency of $10 \mathrm{deg} / \mathrm{s}$.

The numerical simulations are performed for a model of the plant given by Eqs. (30) and (31) based on the CMG described in Sec. VI. The nominal values for the various mass, inertia, center of mass location, and gravitational parameters are given by Eqs. (76-81). Note from Eqs. (80) and (81) that we have considered the case in which there exists a common point that lies on the axis of rotation of each motor, that is, $a=b=c$, so that $G_{i t}=G_{w t}=0$ and $g_{2}=g_{3}=0$, where $g$ is expressed in the inertially fixed frame $\left(\epsilon_{1}, \epsilon_{2}, \epsilon_{3}\right)$ such that, at $t=0,\left(\epsilon_{1}, \epsilon_{2}, \epsilon_{3}\right)$ coincides with $\left(\zeta_{1}, \zeta_{2}, \zeta_{3}\right)$. In this section 


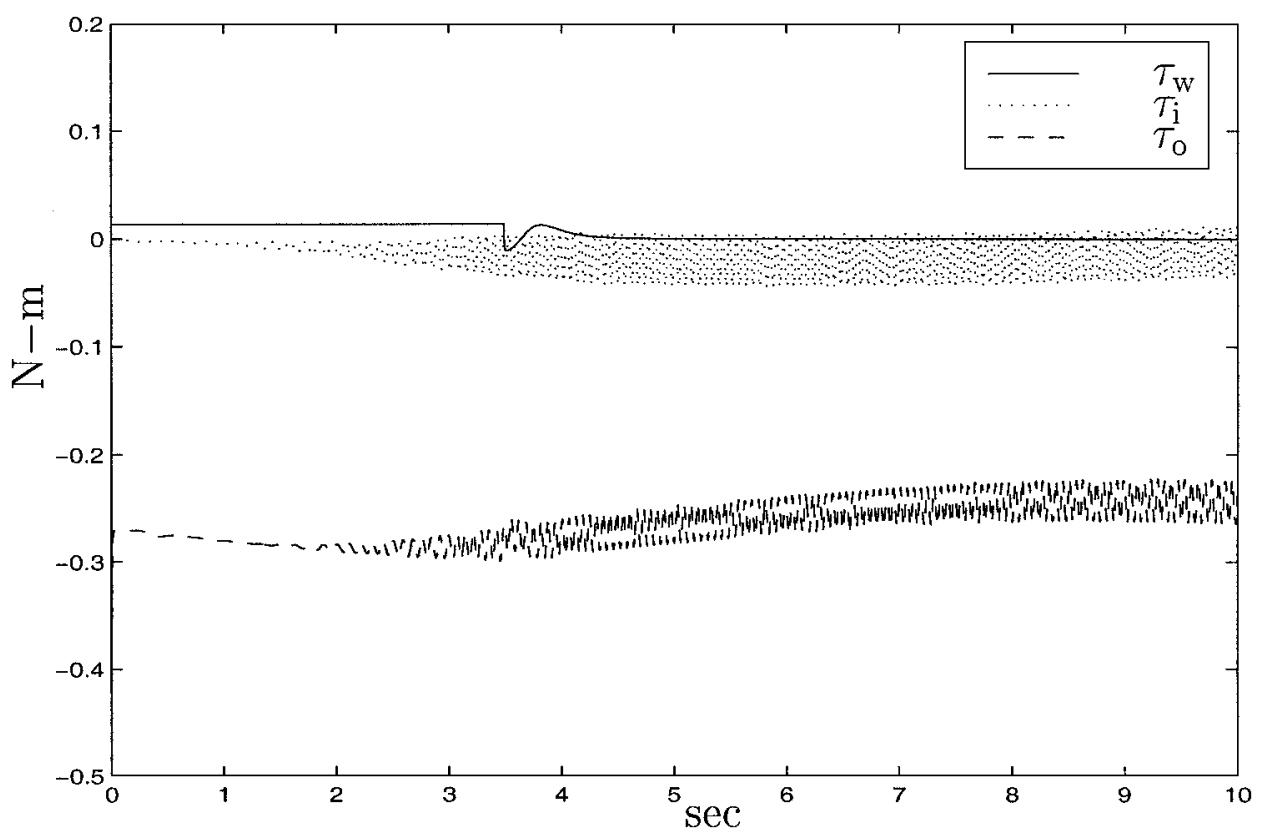

Fig. 4 Motor torques.

we assume that there are no friction and stiffness torques so that $\tilde{F}=\tilde{S}=0$. With this assumption, the number of parameters is reduced from 65 to 21 . In this case, $\alpha=\left[\alpha_{m}^{T} \alpha_{g}^{T}\right]^{T}$, where

$$
\begin{gathered}
\alpha_{m}=\left[\begin{array}{c}
\alpha_{w}^{T} \\
\alpha_{i}^{T} \\
\alpha_{o}^{T}
\end{array}\right], \quad \alpha_{w}=\left[\begin{array}{c}
I_{a 11}^{w} \\
I_{a 22}^{w} \\
I_{a 33}^{w} \\
I_{a 23}^{w} \\
I_{a 13}^{w} \\
I_{a 12}^{w}
\end{array}\right], \quad \alpha_{i}=\left[\begin{array}{c}
I_{b 11}^{i} \\
I_{b 22}^{i} \\
I_{b 33}^{i} \\
I_{b 23}^{i} \\
I_{b 13}^{i} \\
I_{b 12}^{I}
\end{array}\right] \\
\alpha_{o}=I_{c 33}^{o}, \quad\left[\begin{array}{l}
-m_{w} g_{1} \rho_{a r 1} \\
-m_{w} g_{1} \rho_{a t 2} \\
-m_{w} g_{1} \rho_{a t 3} \\
-m_{i} g_{1} \rho_{b u 1} \\
-m_{i} g_{1} \rho_{b u 2} \\
-m_{i} g_{1} \rho_{b u 3} \\
-m_{o} g_{1} \rho_{c v 1} \\
-m_{o} g_{1} \rho_{c v 2}
\end{array}\right]
\end{gathered}
$$

The initial orientation is $q=\left[\begin{array}{lll}0 & 0 & 0\end{array}\right]^{T} \mathrm{rad}$, the initial rate is $\dot{q}=\left[\begin{array}{lll}0 & 0 & 0\end{array}\right]^{T} \mathrm{rad} / \mathrm{s}$, and the initial value of the adaptive parameter is $\hat{\alpha}=\left[\begin{array}{llll}\hat{\alpha}_{w}^{T} & \hat{\alpha}_{i}^{T} & \hat{\alpha}_{o}^{T} & \hat{\alpha}_{g}^{T}\end{array}\right]^{T}$, where $\hat{\alpha}_{w}=1.0 e-3\left[\begin{array}{llll}1.4 & 7.5 & 1.2 & 1.0 e-\end{array}\right.$ $33.0 e-34.0 e-4]^{T} \quad \mathrm{~kg} \cdot \mathrm{m}^{2}, \quad \hat{\alpha}_{i}=1.0 e-4\left[\begin{array}{llll}1.3 & 3.1 & 2.9 & 1.0 e-2\end{array}\right.$ $2.0 e-23.0 e-2]^{T} \mathrm{~kg} \cdot \mathrm{m}^{2}, \hat{\alpha}_{o}=7.2 e-3 \mathrm{~kg} \cdot \mathrm{m}^{2}$ and $\hat{\alpha}_{g}=[3.2 e-2$ $01.2 e-81.2 e-97.2 e-12.0 e-61.0 e-58.1 e-6]^{T} \mathrm{~kg} \cdot \mathrm{m}^{2} / \mathrm{s}^{3}$. The gains are chosen to be

$$
\Lambda(t)=\left[\begin{array}{ccc}
0.2 & 0 & 0 \\
0 & 4 & 0 \\
0 & 0 & 4
\end{array}\right], \quad K(t)=\left[\begin{array}{ccc}
0.1 & 0 & 0 \\
0 & 2.4 & 0 \\
0 & 0 & 2.4
\end{array}\right]
$$

$P=I_{3}$ and $Q=100,000 I_{21}$.

For the CMG described in Sec. VI, the wheel motor can generate a maximum torque of $0.01332 \mathrm{~N} \cdot \mathrm{m}$, the inner gimbal motor can generate a maximum torque of $0.113 \mathrm{~N} \cdot \mathrm{m}$, and the outer gimbal motor can generate a maximum torque of $1.769 \mathrm{~N} \cdot \mathrm{m}$. We apply the control law given by Eqs. (53) and (54) to Eqs. (30) and (31), but saturate the controller so that it does not exceed the maximum allowable torque. We observe from Figs. 2 and 3 that command following is achieved. Figure 4 indicates the control effort required. Figures 5 and 6 indicate the estimates of $\alpha_{w}$, which are given by $\hat{\alpha}_{1}, \ldots, \hat{\alpha}_{6}$. Note from Fig. 5 that $\hat{\alpha}_{1}, \hat{\alpha}_{2}$, and $\hat{\alpha}_{3}$ do not converge to the approximate values as given in Sec. VI and from Fig. 6 that $\hat{\alpha}_{5}$ and $\hat{\alpha}_{6}$ are oscillatory and do not seem to converge.

\section{Experiment Description}

The CMG testbed was designed to allow large angle rotational motion of the wheel. Each of the gimbals is able to rotate nearly $180 \mathrm{deg}$ in both directions, providing a range of motion sufficient for high precession angles and large angle slewing maneuvers. The gimbals cannot complete a full revolution because the electrical connections are made using wires rather than slip rings. The outer gimbal and inner gimbals are machined from a single block of aluminum to provide precise alignment. For details see Ref. 19.

Figure 7 shows a photograph of the actual CMG testbed with connectors and wiring. The wheel shown in Fig. 7 contains slots to which we can add masses to unbalance the wheel. To further vary the center of mass location, the wheel can be moved translationally along its rotation axis.

The approximate values for the various mass, inertia, center of mass location, and center of gravity location parameters based on a nominal configuration of the $\mathrm{CMG}$ are

$$
\begin{gathered}
I_{a}^{w}=\left[\begin{array}{ccc}
4.79 e-4 & 1.0 e-6 & 2.0 e-6 \\
1.0 e-6 & 6.1005 e-4 & 1.0 e-6 \\
2.0 e-6 & 1.0 e-6 & 6.1005 e-4
\end{array}\right] \mathrm{kg} \cdot \mathrm{m}^{2} \\
I_{b}^{i}=\left[\begin{array}{ccc}
1.287 e-3 & 1.0 e-6 & 1.0 e-6 \\
1.0 e-6 & 5.0185 e-4 & 0.0 \\
1.0 e-6 & 0.0 & 1.605 e-3
\end{array}\right] \mathrm{kg} \cdot \mathrm{m}^{2} \\
I_{c 33}^{o}=8.231 e-3 \mathrm{~kg} \cdot \mathrm{m}^{2} \\
m_{1}=-9.81 \mathrm{~m} / \mathrm{s}^{2}, \quad g_{2}=0.0 \mathrm{~m} / \mathrm{s}^{2}, \quad g_{3}=0.0 \mathrm{~m} / \mathrm{s}^{2} \\
\rho_{a t}=\left[\begin{array}{llll}
0.02 & 0.0 & 0.0
\end{array}\right]^{T} \mathrm{~m}, \quad \rho_{b u}=\left[\begin{array}{lll}
-0.07 & 0.0 & 0.0
\end{array}\right]^{T} \mathrm{~m}
\end{gathered}
$$




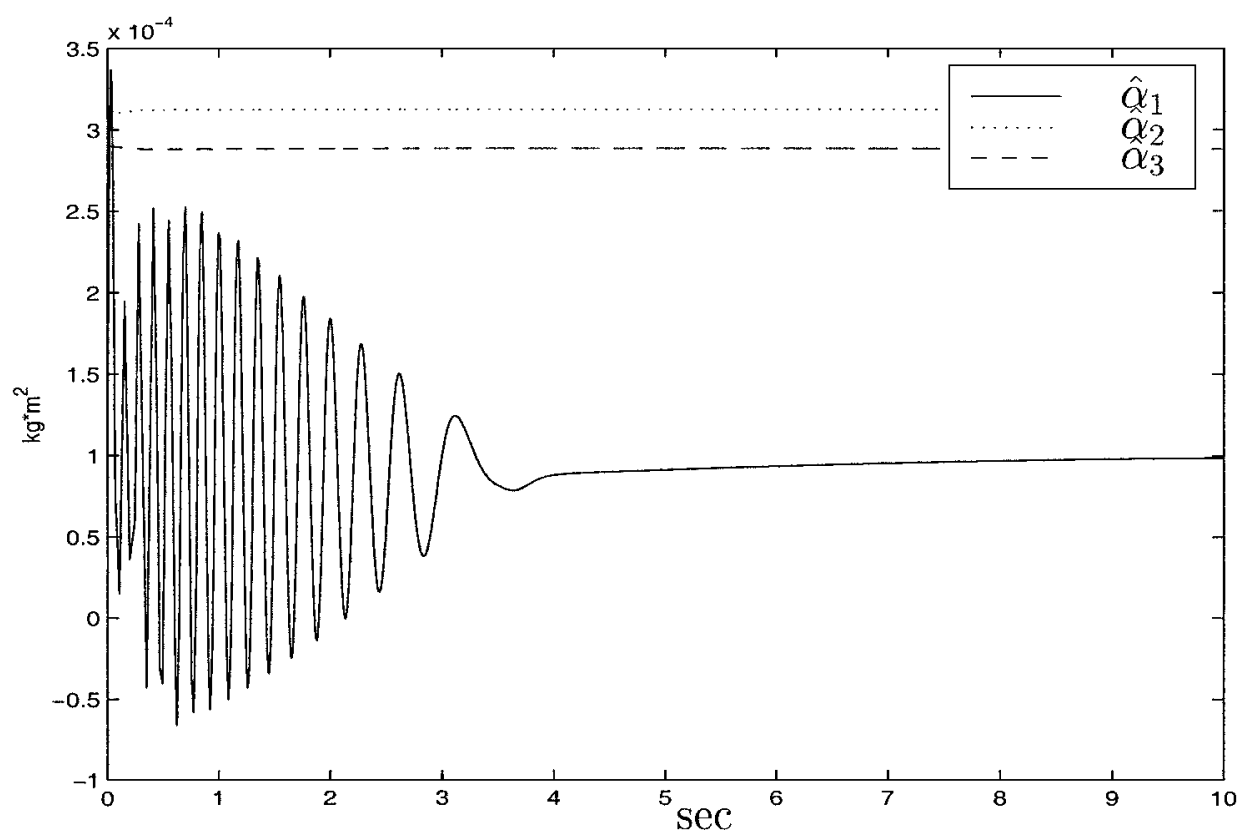

Fig. 5 Adaptive parameters $\hat{\alpha}_{1}, \hat{\alpha}_{2}$, and $\hat{\alpha}_{3}$ (estimates of $I_{a 11}^{w}, I_{a 22}^{w}$, and $I_{a 33}^{w}$, respectively).

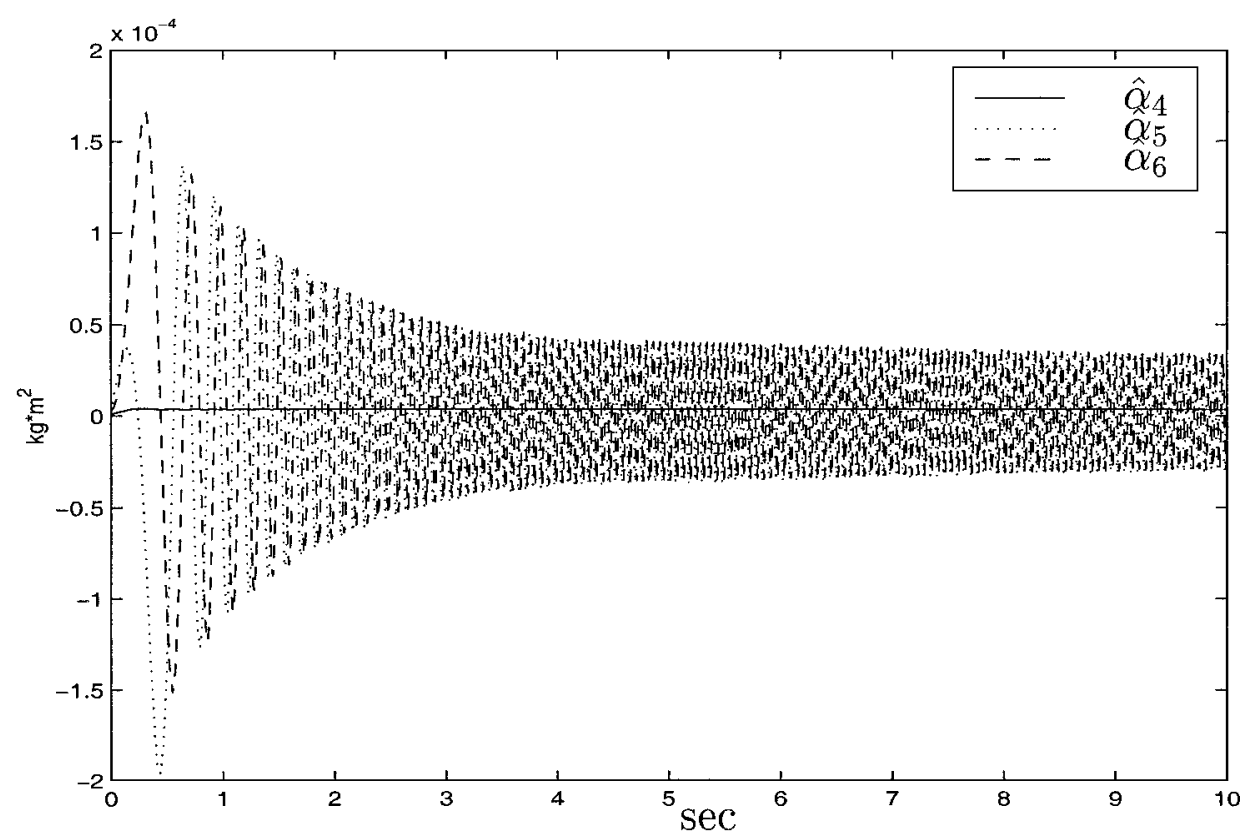

Fig. 6 Adaptive parameters $\hat{\alpha}_{4}, \hat{\alpha}_{5}$, and $\hat{\alpha}_{6}$ (estimates of $I_{a 23}^{w}, I_{a 13}^{w}$, and $I_{a 12}^{w}$, respectively).

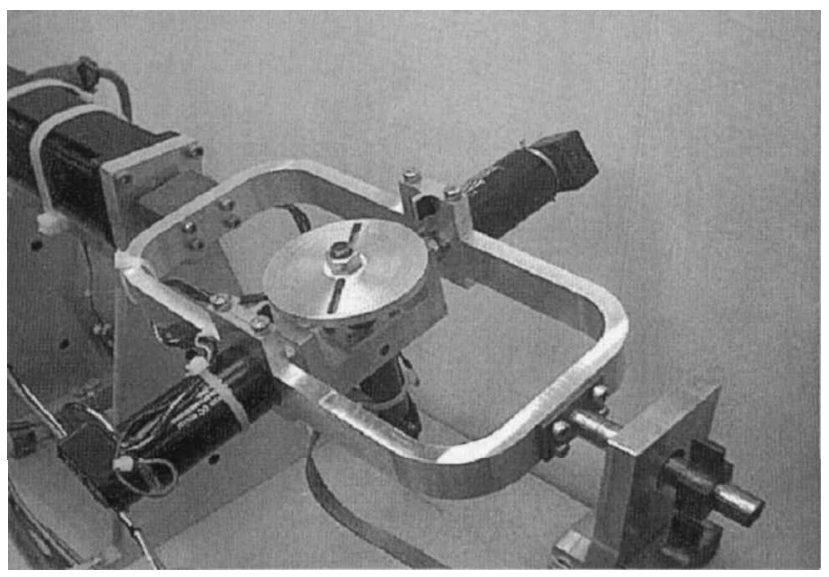

Fig. 7 CMG testbed. where we express $g$ in an inertially fixed frame $\left(\varepsilon_{1}, \varepsilon_{2}, \varepsilon_{3}\right)$ such that, at $t=0,\left(\varepsilon_{1}, \varepsilon_{2}, \varepsilon_{3}\right)$ coincides with $\left(\zeta_{1}, \zeta_{2}, \zeta_{3}\right)$. Note from Eqs. (80) and (81) that in the nominal configuration we have assumed that $a=b=c$.

All of the motors are equipped with optical incremental encoders, providing measurements of the angles of the gimbals and wheel. We differentiate and filter the encoder signals to obtain $\dot{\phi}, \dot{\theta}$, and $\psi$. The inner gimbal and wheel motors, manufactured by Maxon, Inc., were chosen for their high torque-to-weight ratio, low inertia, and low torque ripple. The control processor is the DS1103 board manufactured by dSPACE, Inc. The code for simulation and controller implementation is written in $\mathrm{C}$ using the $\mathrm{S}$-function blocks of Simulink ${ }^{\circledR}$. The sampling rate is $1000 \mathrm{~Hz}$.

\section{Experimental Results}

In this section we present experimental results to illustrate command following for the desired trajectory 


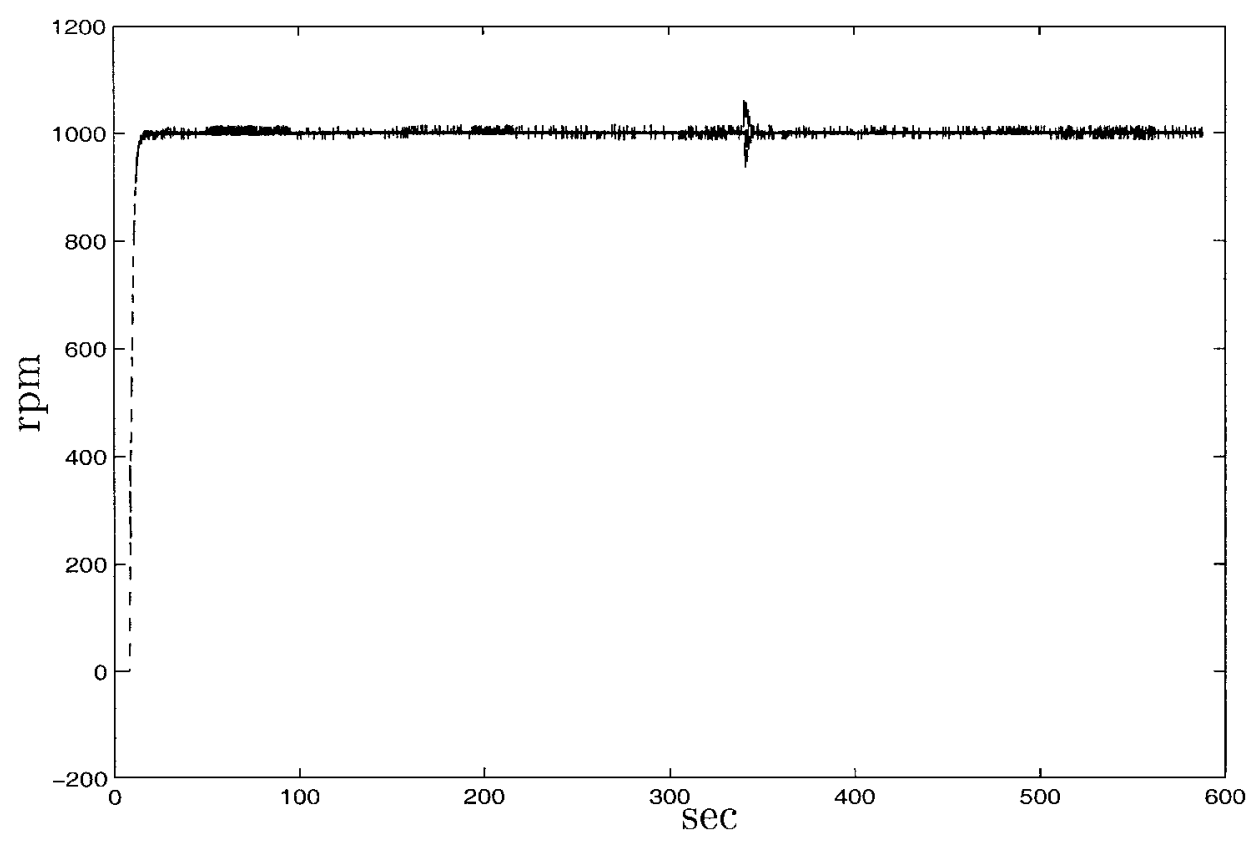

Fig. 8 Wheel rate.

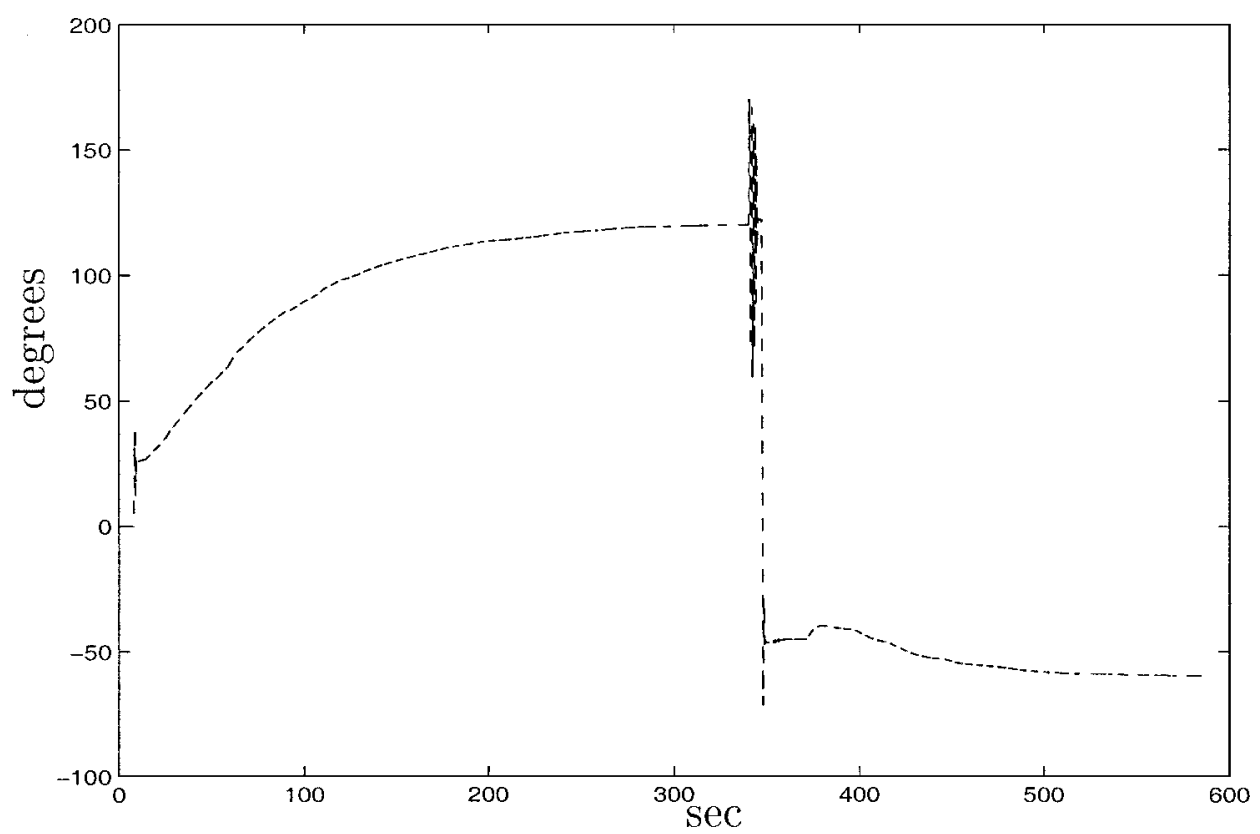

Fig. 9 Inner gimbal angle.

$$
\begin{aligned}
\phi_{d}(t) & =2000 \pi / 60 t \mathrm{rad} \\
\theta_{d}(t) & =120 \pi / 180 \mathrm{rad} \\
\psi_{d}(t) & =-40 \pi / 180 \mathrm{rad}
\end{aligned}
$$

This command represents a CMG motion in which the wheel spins at a constant rate of $1000 \mathrm{rpm}$, the inner gimbal is oriented to an angle of $120 \mathrm{deg}$, and the outer gimbal is oriented to an angle of $-40 \mathrm{deg}$. Once convergence has been attained, the command is abruptly changed so that the inner gimbal is reoriented to an angle of $-60 \mathrm{deg}$ and the outer gimbal is reoriented to an angle of $60 \mathrm{deg}$. The control law given by Eqs. (53) and (54) is applied to the CMG described in Sec. VI.

We assume the friction and stiffness torques are of the form

$$
F(p, \hat{p})=\left[\begin{array}{ccc}
F_{1} & 0 & 0 \\
0 & F_{2} & 0 \\
0 & 0 & F_{3}
\end{array}\right]
$$

where $F_{1}, F_{2}$, and $F_{3}$ are real numbers independent of $p$ and $\hat{p}$,

$$
S(p)=\left[\begin{array}{ccc}
s_{1} \sin \left(p_{1}\right) & 0 & 0 \\
0 & s_{2} \sin \left(p_{2}\right) & 0 \\
0 & 0 & s_{3} \sin \left(p_{3}\right)
\end{array}\right], \quad s_{1}, s_{2}, s_{3} \in \Re
$$

so that $\alpha=\left[\begin{array}{llll}\alpha_{m}^{T} & \alpha_{g}^{T} & \alpha_{f}^{T} & \alpha_{s}^{T}\end{array}\right]^{T}$, where $\alpha_{m}$ and $\alpha_{g}$ are given by Eq. (74), and

$$
\alpha_{f}=\left[\begin{array}{l}
f_{1} \\
f_{2} \\
f_{3}
\end{array}\right], \quad \alpha_{s}=\left[\begin{array}{l}
s_{1} \\
s_{2} \\
s_{3}
\end{array}\right]
$$

The tuning parameters are chosen to be

$$
\Lambda(t)=\left[\begin{array}{ccc}
50.0 & 0 & 0 \\
0 & 0.2 & 0 \\
0 & 0 & 0.2
\end{array}\right]+\left(1-e^{-0.001 t}\right)\left[\begin{array}{ccc}
-40.0 & 0 & 0 \\
0 & 1.8 & 0 \\
0 & 0 & 19.8
\end{array}\right]
$$




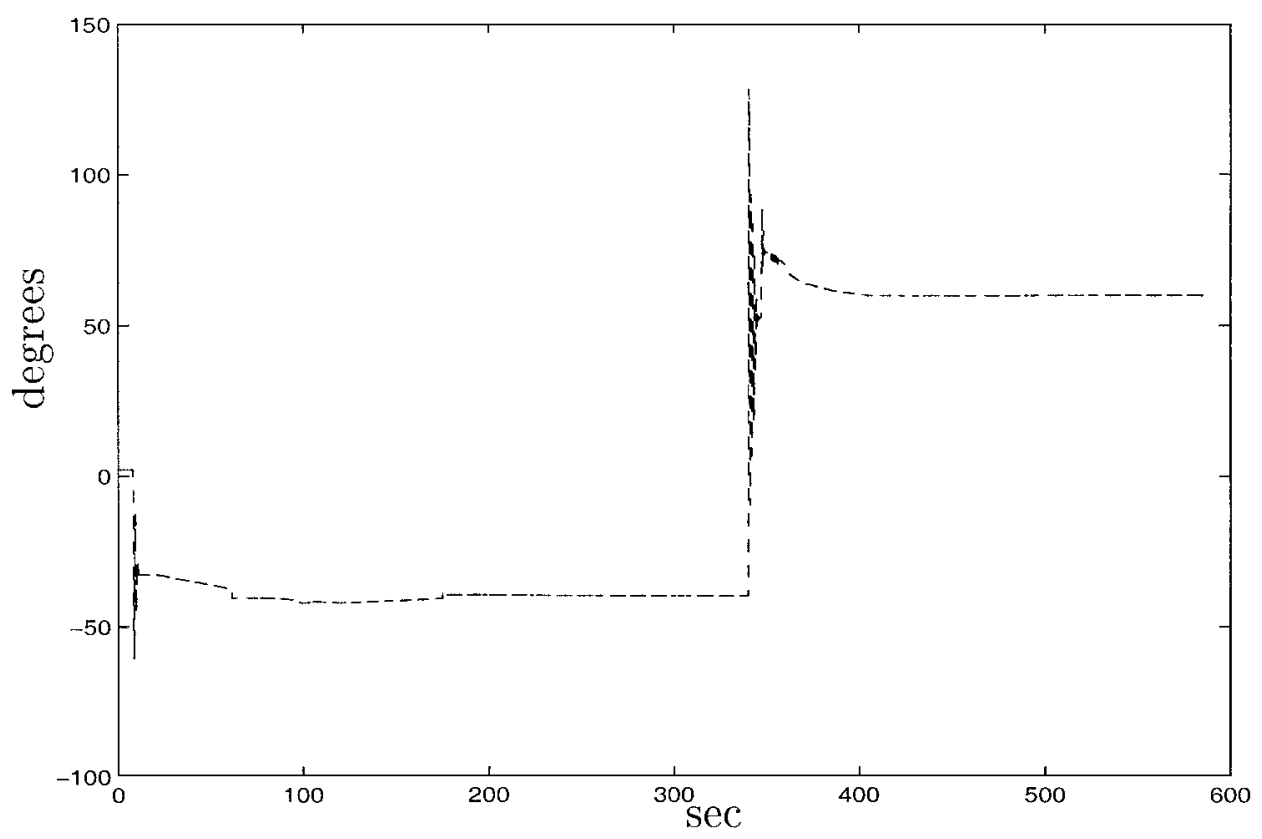

Fig. 10 Outer gimbal angle.

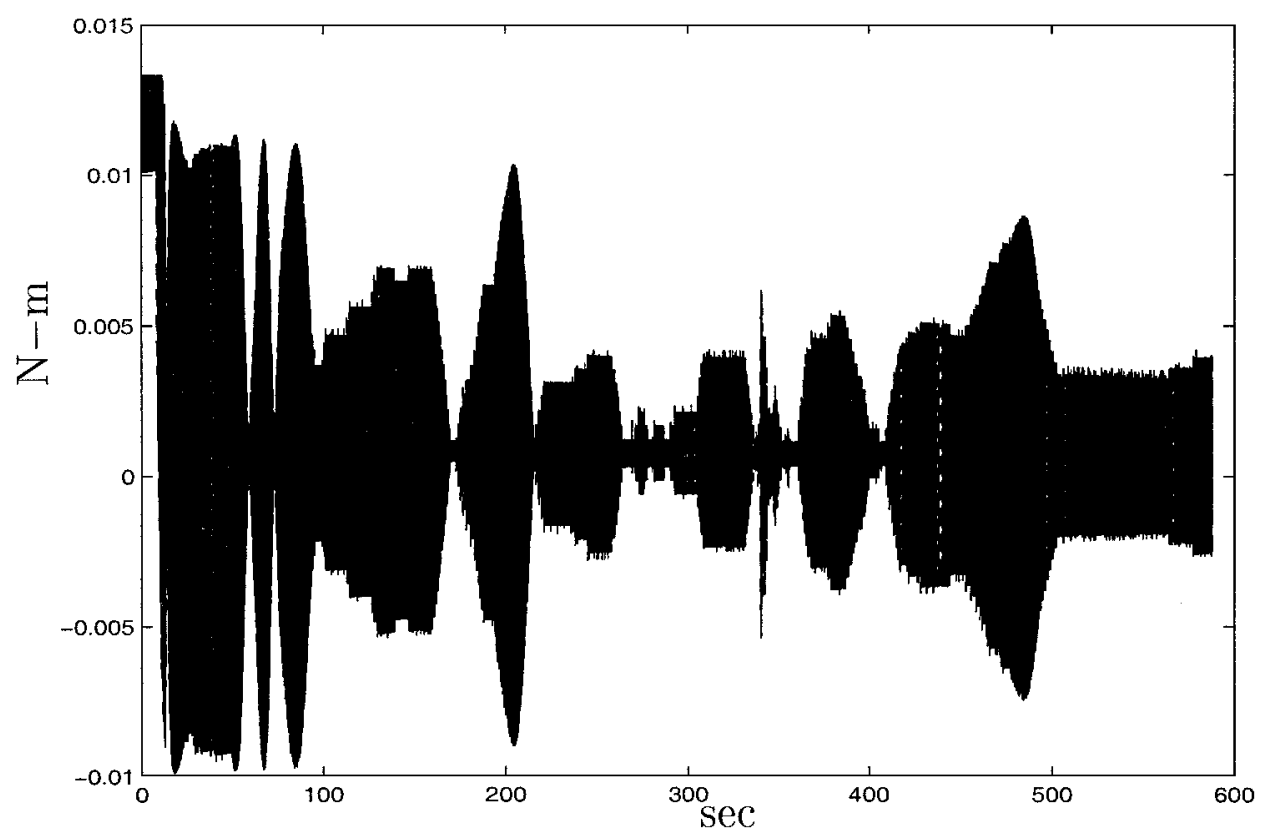

Fig. 11 Wheel motor torque.

$$
\begin{aligned}
K(t) & =\left[\begin{array}{ccc}
2.0 e-4 & 0 & 0 \\
0 & 4.0 e-5 & 0 \\
0 & 0 & 4.0 e-4
\end{array}\right] \\
& +\left(1-e^{-0.001 t}\right)\left[\begin{array}{ccc}
0.0 & 0 & 0 \\
0 & 0.0 & 0 \\
0 & 0 & 0.0396
\end{array}\right]
\end{aligned}
$$

$P=1.0 e-3 I_{3}$

$Q=\operatorname{diag}\left(Q_{1}, Q_{2}, Q_{3}, Q_{4}, Q_{5}, Q_{6}, Q_{7}, Q_{8}\right)$

where

$Q_{1}=\operatorname{diag}(1.0 e-11,1.0 e-11,1.0 e-11,1.0 e-14$,

$$
1.0 e-14,1.0 e-14)
$$

$Q_{2}=\operatorname{diag}(1.0 e-6,1.0 e-6,1.0 e-6,1.0 e-9$,

$$
1.0 e-9,1.0 e-9), \quad Q_{3}=1.0 e-2
$$

$$
\begin{aligned}
Q_{4} & =1.0 e-5 I_{3}, \quad Q_{5}=1.0 e-2 I_{3}, \quad Q_{6}=1.0 e-3 I_{2} \\
Q_{7} & =\operatorname{diag}(1.0 e-9,1.0 e-5,1.0 e-1) \\
Q_{8} & =\operatorname{diag}(1.0 e-4,100.0,10.0)
\end{aligned}
$$

The gains were chosen to prevent saturation of the motors for any appreciable period of time. In our tests on our setup, we found that saturation of the motors for significant periods of time resulted in the buildup of large amplitude oscillations. Also, saturation of the motors might cause damage to the motors if continued for long periods of time. With this view in mind, most of the time-varying gains are initially chosen small because parametric uncertainity is initally large. As the adaptation proceeds, these gains are increased. However, note that $\Lambda_{11}(t)$ actually decreases in magnitude.

The torques that are transmitted to the gimbals and wheel can be turned on or off using a master switch that can be controlled using software developed by dSPACE, Inc. We apply the control law (53) and (54) at $t=0 \mathrm{~s}$, but the master switch is turned on 


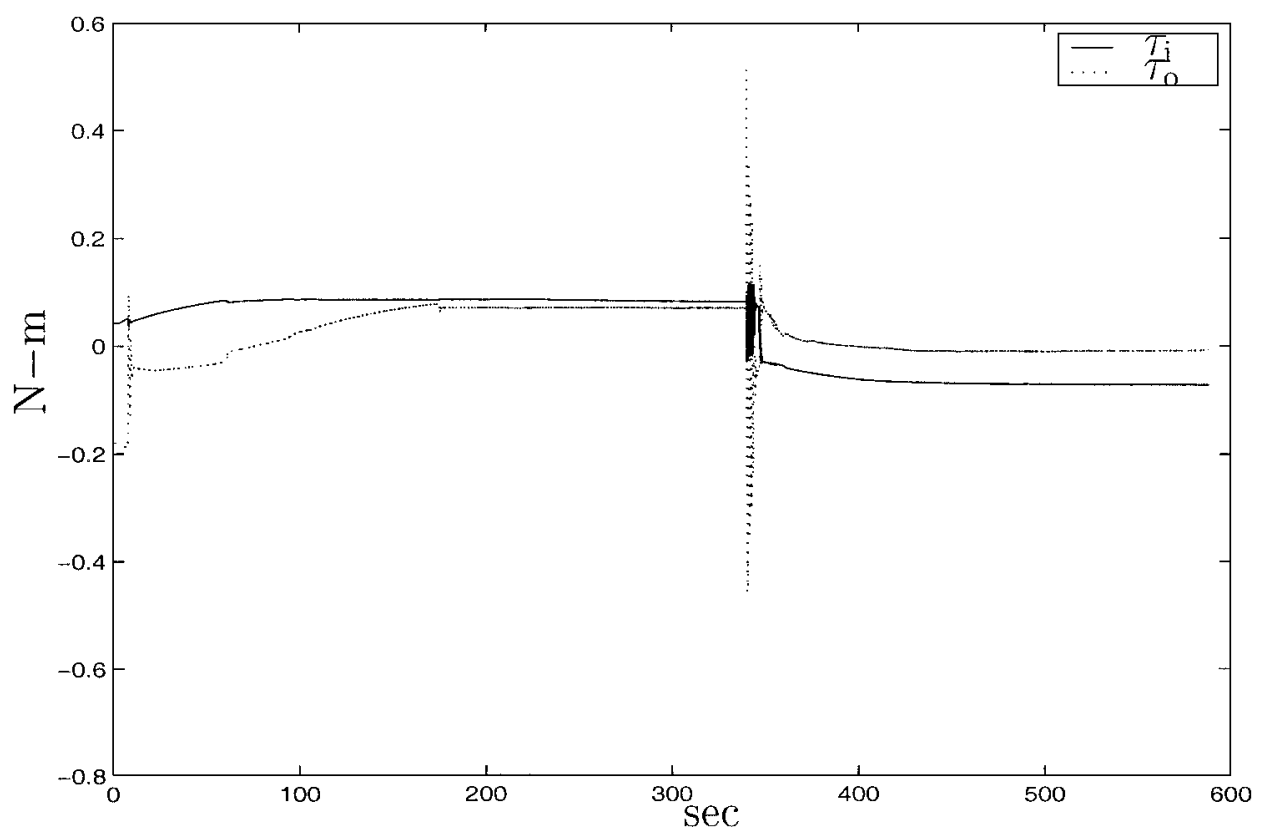

Fig. 12 Torque generated by inner and outer gimbal motors.

only at approximately $t=20 \mathrm{~s}$, and thus, the motors are effectively turned off for $t \in[0,20)$. The spikes in Figs. 8-11 at approximately $t=20 \mathrm{~s}$ are due to the master switch being turned on. Figures 11 and 12 show the controlefforts as required by the control law. Before $t=20 \mathrm{~s}$, these torques are not transmitted to the CMG because the master switch has not yet been turned on.

We observe from Fig. 8 that the wheel attains a speed of $1000 \mathrm{rpm}$ at approximately $t=30 \mathrm{~s}$. Figure 9 shows that the inner gimbal attains an angle of $120 \mathrm{deg}$ at approximately $t=350 \mathrm{~s}$, and Fig. 10 shows that the outer gimbal attains an angle of $-40 \mathrm{deg}$ at approximately $t=200 \mathrm{~s}$. At approximately $t=350 \mathrm{~s}$, we modify the command as described earlier. We observefrom Fig. 8 that the wheel attains the speed of $1000 \mathrm{rpm}$ at approximately $t=350 \mathrm{~s}$, from Fig. 9 that the inner gimbal attains an angle of $-60 \mathrm{deg}$ at approximately $t=600 \mathrm{~s}$, and from Fig. 10 that the outer gimbal attains an angle of $60 \mathrm{deg}$ at approximately $t=400 \mathrm{~s}$. In Figs. 9 and 10, it can be seen that the gimbals undergo transients due to startup as well as a transient at $t=350 \mathrm{~s}$ due to the abrupt change in setpoint.

\section{Conclusions}

In this paper, we are interested in developing a control algorithm that follows a commanded CMG rotational motion, including commanded rotational motions that are unbounded. To do this, we describe the rotational motion of the CMG in terms of the trigonometric functions of the half-angles of the gimbals and wheel. This formulation transforms unbounded rotational motion of the CMG onto motion on a compact set and is the key ingredient in the development of the control algorithm (53) and (54).

In a similar vein, it is the use of time-varyinggains that permits the successful use of Eqs. (53) and (54) to achieve command following in our experimental setup. The use of constant gains resulted in either saturation of the controller for significant periods of time, which led to the buildup of large amplitude oscillations, or to extremely slow time responses.

In future research the control law will be modified to suppress reaction torques transmitted to the support structure due to imbalance.

\section{References}

${ }^{1}$ Bryson, A. E., Jr., Control of Spacecraft and Aircraft, Princeton Univ. Press, Princeton, NJ, 1994, pp. 74-92.

${ }^{2}$ Marguiles, G., and Aubrun, J. N., "Geometric Theory of Single-Gimbal Control Moment Gyro Systems," AIAA Guidance and Control Conference,
AIAA, New York, 1976, pp. 255-267.

${ }^{3}$ Liden, S. P., "Precision CMG Control for High-Accuracy Pointing," AIAA Guidance and Control Conference, AIAA, New York, 1973, pp. 236240.

${ }^{4}$ Chubb, W. B., Kennel, H. F., Rupp, C. C., and Seltzer, S. M., "Flight Performance of Skylab Attitude and Pointing Control System," AIAA Mechanics and Control of Flight Conference, AIAA, New York, 1974, pp. 220227.

${ }^{5}$ Kurokawa, H., Yajima, N., and Usui, S., "A New Steering Law of a Single Gimbal CMG System of Pyramid Configuration," IFAC Automatic Control in Space, IEEE Publications, Piscataway, NJ, 1985, pp. 251-257.

${ }^{6}$ Bodora, J. A., and Bamlde, H., "Experimental and System Study of Reaction Wheels," ESA Contract Report, 1982.

${ }^{7}$ Neat, G. W., Melody, J. W., and Lurie, B. J., "Vibration Attenuation Approach for Spaceborne Optical Interferometers," IEEE Transactions on Control Systems Technology, Vol. 6, No. 6, 1998, pp. 687-700.

${ }^{8}$ Greenwood, D. T., Principles of Dynamics, Prentice-Hall, Englewood Cliffs, NJ, 1988, pp. 239-299.

${ }^{9}$ Bayard, D. S., and Wen, T. J., "A New Class of Control Laws for Robotic Manipulators-Part II: Adaptive Case," International Journal of Control, Vol. 47, No. 5, 1988, pp. 1387-1406.

${ }^{10}$ Arimoto, S., Control Theory of Non-Linear Mechanical Systems: A Passivity-Based and Circuit-Theoretic Approach, Vol. 49, Oxford Univ. Press, Oxford, England, U.K., 1996.

${ }^{11}$ Koditschek, D. E., "Appplication of a New LyapunovFunction to Global Adaptive Attitude Tracking," IEEE Conference on Decision and Control, Inst. of Electrical and Electronics Engineers, New York, 1988, pp. 63-68.

${ }^{12}$ Hale, J. K., Ordinary Differential Equations, Wiley, New York, 1969, p. 305 .

${ }^{13}$ Slotine, J. J. E., and Li, W., Applied Nonlinear Control, Prentice-Hall, Upper Saddle River, NJ, 1989.

${ }^{14}$ Sastry, S., and Bodson, M., Adaptive Control: Stability, Convergence, and Robustness, Prentice-Hall, Upper Saddle River, NJ, 1989.

${ }^{15}$ Astrom, K. J., and Wittenmark, B., Adaptive Control, 2nd ed., AddisonWesley, Reading, MA, 1995.

${ }^{16}$ Narendra, K. S., and Annaswamy, A. M., Stable Adaptive Systems, Prentice Hall, Englewood Cliffs, NJ, 1988, Chap. 1.

${ }^{17}$ Ahmed, J., Coppola, V. T., and Bernstein, D. S., "Asymptotic Tracking of Spacecraft Attitude Motion with Inertia Identification," Journal of Guidance, Control, and Dynamics, Vol. 21, No. 5, 1998, pp. 684-691.

${ }^{18}$ Khalil, H. K., Nonlinear Systems, Prentice-Hall, Upper Saddle River, NJ, 1996, p. 77, 138.

${ }^{19}$ Ahmed, J., Miller, R. H., Hoopman, E. H., Coppola, V. T., Andrusiak, T., Acton, D., and Bernstein, D. S., "An Actively Controlled Control Moment Gyro/GyroPendulum Testbed," Proceedings of Conference on Control Applications, IEEE Publications, Piscataway, NJ, 1997, pp. 250-252. 